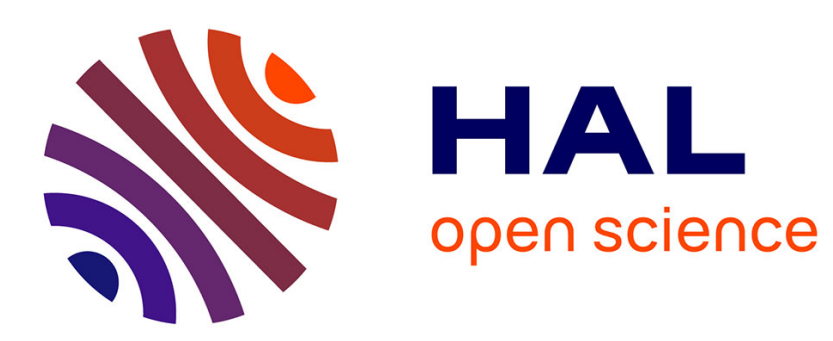

\title{
An obstruction to small time local null controllability for a viscous Burgers' equation
}

Frédéric Marbach

\section{To cite this version:}

Frédéric Marbach. An obstruction to small time local null controllability for a viscous Burgers' equation. Annales Scientifiques de l'École Normale Supérieure, 2018, 51 (5), pp.1129-1177. 10.24033/asens.2373 . hal-01229493

\section{HAL Id: hal-01229493 \\ https://hal.science/hal-01229493}

Submitted on 16 Nov 2015

HAL is a multi-disciplinary open access archive for the deposit and dissemination of scientific research documents, whether they are published or not. The documents may come from teaching and research institutions in France or abroad, or from public or private research centers.
L'archive ouverte pluridisciplinaire HAL, est destinée au dépôt et à la diffusion de documents scientifiques de niveau recherche, publiés ou non, émanant des établissements d'enseignement et de recherche français ou étrangers, des laboratoires publics ou privés. 


\title{
An obstruction to small time local null controllability for a viscous Burgers' equation
}

\author{
Frédéric Marbach *
}

November 16, 2015

\begin{abstract}
In this work, we are interested in the small time local null controllability for the viscous Burgers' equation $y_{t}-y_{x x}+y y_{x}=u(t)$ on the line segment [0,1], with null boundary conditions. The second-hand side is a scalar control playing a role similar to that of a pressure. In this setting, the classical Lie bracket necessary condition $\left[f_{1},\left[f_{1}, f_{0}\right]\right]$ introduced by Sussmann fails to conclude. However, using a quadratic expansion of our system, we exhibit a second order obstruction to small time local null controllability. This obstruction holds although the information propagation speed is infinite for the Burgers equation. Our obstruction involves the weak $H^{-5 / 4}$ norm of the control $u$. The proof requires the careful derivation of an integral kernel operator and the estimation of residues by means of weakly singular integral operator estimates.
\end{abstract}

\section{Introduction}

\subsection{Description of the system and our main result}

For $T>0$ a small positive time, we consider the line segment $x \in[0,1]$ and the following onedimensional viscous Burgers' controlled system:

$$
\left\{\begin{aligned}
y_{t}-y_{x x}+y y_{x} & =u(t) & & \text { in }(0, T) \times(0,1), \\
y(t, 0) & =0 & & \text { in }(0, T), \\
y(t, 1) & =0 & & \text { in }(0, T), \\
y(0, x) & =y_{0}(x) & & \text { in }(0,1) .
\end{aligned}\right.
$$

The scalar control $u \in L^{2}(0, T)$ plays a role somewhat similar to that of a pressure for multidimensional fluid systems. Unlike some other studies, our control term $u$ depends only on time and not on the space variable. It is supported on the whole segment $[0,1]$. For any initial data $y_{0} \in H_{0}^{1}(0,1)$ and any fixed control $u \in L^{2}(0, T)$, it can be shown (see Lemma 8 below) that system (1.1) has a unique solution in the space $X_{T}=L^{2}\left((0, T) ; H^{2}(0,1)\right) \cap H^{1}\left((0, T) ; L^{2}(0,1)\right)$. We are interested in the behavior of this system in the vicinity of the null equilibrium state.

Definition 1. We say that system (1.1) is small time locally null controllable if, for any small time $T>0$, for any small size of the control $\eta>0$, there exists a region $\delta>0$ such that:

$$
\forall\left|y_{0}\right|_{H_{0}^{1}} \leq \delta, \exists u \in L^{2}(0, T),|u|_{2} \leq \eta \text { such that } y(T, \cdot)=0,
$$

where $y \in X_{T}$ is the solution to system (1.1) with initial condition $y_{0}$ and control $u$.

Theorem 1. System (1.1) is not small time locally null controllable. Indeed, there exist $T, \eta>0$ such that, for any $\delta>0$, there exists $y_{0} \in H_{0}^{1}(0,1)$ with $\left|y_{0}\right|_{H_{0}^{1}} \leq \delta$ such that, for any control $u \in L^{2}(0, T)$ with $|u|_{2} \leq \eta$, the solution $y \in X_{T}$ to (1.1) satisfies $y(T, \cdot) \neq 0$.

We will see in the sequel that our proof actually provides a stronger result. Indeed, we prove that, for small times and small controls, whatever the small initial data $y_{0}$, the state $y(t)$ drifts towards a fixed direction. Of course, this prevents small time local null controllability as a direct consequence.

\footnotetext{
*Email: frederic.marbach@upmc.fr. Address: Laboratoire Jacques-Louis Lions, Université Pierre et Marie Curie, Institut Universitaire de France, 4, Place Jussieu, 75252 Paris Cedex, France. Work supported by the ERC advanced grant 266907 (CPDENL) of the 7th Research Framework Programme (FP7)
} 


\subsection{Motivation: small time obstructions despite infinite propagation speed}

As an example, let us consider the following transport control system:

$$
\left\{\begin{aligned}
y_{t}+M y_{x} & =0 & & \text { in }(0, T) \times(0, L), \\
y(t, 0) & =v_{0}(t) & & \text { in }(0, T), \\
y(0, x) & =y_{0}(x) & & \text { in }(0, L),
\end{aligned}\right.
$$

where $T>0$ is the total time, $M>0$ the propagation speed and $L>0$ the length of the domain. The control is the boundary data $v_{0}$. No condition is imposed at $x=1$ since the characteristics flow out of the domain. For system (1.3), small time local null controllability cannot hold. Indeed, even if the initial data $y_{0}$ is very small, the control is only propagated towards the right at speed $M$. Thus, if $T<L / M$, controllablity does not hold. Of course, if $T \geq L / M$, the characteristics method allows to construct an explicit control to reach any final state $y_{1}$ at time $T$. In this context, the obstruction to controllability comes from the fact that the information propagation speed is bounded. Indeed, let us modify slightly system (1.3) into:

$$
\left\{\begin{aligned}
y_{t}-\nu y_{x x}+M y_{x} & =0 & & \text { in }(0, T) \times(0, L), \\
y(t, 0) & =v_{0}(t) & & \text { in }(0, T), \\
y(t, 1) & =0 & & \text { in }(0, T), \\
y(0, x) & =y_{0}(x) & & \text { in }(0, L),
\end{aligned}\right.
$$

where $\nu>0$ is a (very small) viscosity. This system is small time globally null controllable, for any $\nu>0$. Of course, the cost of controllablity must explode as $\nu \rightarrow 0$ if $T$ is too small (see [22] for a precise study of the cost of controllability for (1.4)). What we want to underline here, is that the infinite information propagation speed yields (at least in this context) small time local controllability.

Therefore, there is a strong interest for systems where small time local controllability does not hold despite an infinite information propagation speed.

An example of such a system is the control of a quantum particle in a moving potential well (box). This is a bilinear controllability problem for the Schrödinger equation. For such system, it can be shown that large time controllablity holds (see [4] if only the particle needs to be controlled or [5] to control both the particle and the box). For small times, negative results have been obtained by Coron in [18] (when one tries to control both the particle and the position of the box), by Beauchard, Coron and Teissman in [6] for large controls (but smooth potentials) and by Beauchard and Morancey in [7] (under an assumption corresponding to a Lie-bracket condition $\left[f_{1},\left[f_{1}, f_{0}\right]\right] \neq 0$ ). This last paper is related to ours since their proof relies on a coercivity estimate involving the $H^{-1}$ norm of the control. This is natural as we will see in paragraph 1.5. We refer the reader to these papers for more details and surveys on the controllability of Schrödinger equations.

Theorem 1 can be seen as another example of a situation (in the context of fluid dynamics) where small time local controllability fails despite an infinite propagation speed.

\subsection{Previous works concerning Burgers' controllability}

Let us recall known results concerning the controllability of the viscous Burgers' equation. More generally, we introduce the following system:

$$
\left\{\begin{aligned}
y_{t}-y_{x x}+y y_{x}=u(t) & \text { in }(0, T) \times(0,1), \\
y(t, 0)=v_{0}(t) & \text { in }(0, T), \\
y(t, 1)=v_{1}(t) & \text { in }(0, T), \\
y(0, x)=y_{0}(x) & \text { in }(0,1),
\end{aligned}\right.
$$

where $v_{0}$ and $v_{1}$ are seen as additional controls with respect to the single control $u$ of system (1.1). Various settings have been studied (with either one or two boundary controls, with or without $u$ ). Once again, here $u$ only depends on $t$ and not on $x$. Some studies have been carried out with $v_{0}=v_{1}=0$ and a source term $u(t, x) \chi_{[a, b]}$ for $0<a<b<1$. However, these studies are equivalent to boundary controls thanks to the usual domain extension argument. Up to our knowledge, Theorem 1 is the first result concerning the case without any boundary control and a scalar control $u$. 
We start with results involving only a single boundary control (either $v_{0}$ or $v_{1}$ by symmetry) and $u=0$.

In [31], Fursikov and Imanuvilov prove small time local controllability in the vicinity of trajectories of system (1.5). Their proof relies on Carleman estimates for the parabolic problem obtained by seeing the non-linear term $y y_{x}$ as a small forcing term.

Global controllability towards steady states of system (1.5) is possible in large time. Such studies have been carried out by Fursikov and Imanuvilov in [30] for large time global controllability towards all steady states, and by Coron in [20] for global null controllability in bounded time (ie. bounded independently on the initial data).

However, small time global controllability does not hold. The first obstruction was obtained by Diaz in [24]. He gives a restriction for the set of attainable states starting from 0 . Indeed, they must lie under some limit state corresponding to an infinite boundary control $v_{1}=+\infty$.

Fernández-Cara and Guerrero derived an asymptotic of the minimal null-controllability time $T(r)$ for initial states of $H^{1}$ norm lower than $r$ (see [25]). This shows that the system is not small time globally null controllable.

We move on to two boundary controls $v_{0}$ and $v_{1}$, still with $u=0$. Guerrero and Imanuvilov prove in [32] that neither small time null controllability nor bounded time global controllability hold in this context. Hence, controlling the whole boundary does not provide better controllability properties.

When three scalar controls (namely $u(t), v_{0}$ and $v_{1}$ ) are used, Chapouly has shown in [17] that the system is small time globally exactly controllable to the trajectories. Her proof relies on the return method and on the fact that the corresponding inviscid Burgers' system is small time exactly controllable (see [19, Chapter 6] for other examples of this method applied to Euler or Navier-Stokes).

When $v_{1}=0$, but $u$ and $v_{0}$ are controlled, the author proved in [35] that small time global null controllability holds. Indeed, although a boundary layer appears near the uncontrolled part of the boundary at $x=1$, precise estimation of the creation and dissipation of the boundary layer allows to conclude.

Concerning the controllability of the inviscid Burgers' equation, some works have be carried out. In [2], Ancona and Marson describe the set of attainable states in a pointwise way for the Burgers' equation on the half-line $x \geq 0$ with only one boundary control at $x=0$. In [33], Horsin describes the set of attainable states for a Burgers' equation on a line segment with two boundary controls. Thorough studies are also carried out in [1] by Adimurthi et al. In [39], Perrollaz studies the controllability of the inviscid Burgers' equation in the context of entropy solutions with the additional control $u(\cdot)$ and two boundary controls.

\subsection{A quadratic approximation for the non-linear system}

Starting now, we introduce $\varepsilon=T$ to remember that the total allowed time for controllability is small. Moreover, we want to use the well-known scaling trading small time with small viscosity for viscous fluid equations. Therefore, we introduce, for $t \in(0,1)$ and $x \in(0,1), \tilde{y}(t, x)=\varepsilon y(\varepsilon t, x)$. Hence, $\tilde{y}$ is the solution to:

$$
\left\{\begin{aligned}
\tilde{y}_{t}-\varepsilon \tilde{y}_{x x}+\tilde{y} \tilde{y}_{x} & =\tilde{u}(t) & & \text { in }(0,1) \times(0,1), \\
\tilde{y}(t, 0) & =0 & & \text { in }(0,1), \\
\tilde{y}(t, 1) & =0 & & \text { in }(0,1), \\
\tilde{y}(0, x) & =\tilde{y}_{0}(x) & & \text { in }(0,1),
\end{aligned}\right.
$$

where $\tilde{u}(t)=\varepsilon^{2} u(\varepsilon t)$ and $\tilde{y}_{0}=\varepsilon y_{0}$. This scaling is widely used for controllability results since small viscosity developments are easier to handle. As we will prove in section 6 , system (1.6) can help us to deduce results for system (1.1). To further simplify the computations in the following sections, let us drop the tilda signs and the initial data. Therefore, we will study the behavior of the following 
system near $y \equiv 0$ :

$$
\left\{\begin{aligned}
y_{t}-\varepsilon y_{x x}+y y_{x}=u(t) & & \text { in }(0,1) \times(0,1), \\
y(t, 0)=0 & & \text { in }(0,1), \\
y(t, 1)=0 & & \text { in }(0,1), \\
y(0, x)=0 & & \text { in }(0,1) .
\end{aligned}\right.
$$

Properties proven on system (1.7) will easily be translated into properties for system (1.1) in Section 6 . Moreover, since we are studying local null controllability, both the control $u$ and the state $y$ are small. Thus, if $\eta$ describes the size of the control as in Definition 1, let us name our control $\eta u(t)$, with $u$ of size $\mathcal{O}(1)$. We expand $y$ as $y=\eta a+\eta^{2} b+\mathcal{O}\left(\eta^{3}\right)$, and we compute the associated systems:

$$
\left\{\begin{aligned}
a_{t}-\varepsilon a_{x x} & =u(t) & & \text { in }(0,1) \times(0,1), \\
a(t, 0) & =0 & & \text { in }(0,1), \\
a(t, 1) & =0 & & \text { in }(0,1), \\
a(0, x) & =0 & & \text { in }(0,1)
\end{aligned}\right.
$$

and

$$
\left\{\begin{aligned}
b_{t}-\varepsilon b_{x x} & =-a a_{x} & & \text { in }(0,1) \times(0,1), \\
b(t, 0) & =0 & & \text { in }(0,1), \\
b(t, 1) & =0 & & \text { in }(0,1), \\
b(0, x) & =0 & & \text { in }(0,1) .
\end{aligned}\right.
$$

It is easy to see that system (1.8) is not controllable. Indeed, the control $u(t)$ can actually be written as $u(t) \chi_{[0,1]}$, and $\chi_{[0,1]}$ is an even function on the line segment $[0,1]$. Thus, the control only acts on even modes of $a$. In the linearized system (1.8), all odd modes evolve freely. This motivates the second order expansion of our Burgers' system in order to understand its controllability properties using $b$. Given systems (1.8) and (1.9), we know that $a$ is even and $b$ is odd.

\subsection{A finite dimensional counterpart}

Systems (1.8) and (1.9) exhibit an interesting structure. Indeed, the first system is fully controllable (if we consider that $a$ lives within the subspace of even functions), while the second system is indirectly controlled through a quadratic form depending on $a$. Let us introduce the following finite dimensional control system:

$$
\left\{\begin{array}{l}
\dot{a}=M a+u(t) m \quad \text { in }(0, T) \\
\dot{b}=L b+Q(a, a) \text { in }(0, T)
\end{array}\right.
$$

where the states $a(t), b(t) \in \mathbb{R}^{n} \times \mathbb{R}^{p}, M$ is an $n \times n$ matrix, $m$ is a fixed vector in $\mathbb{R}^{n}$ along which the scalar control acts, $L$ is a $p \times p$ matrix and $Q$ is a quadratic function from $\mathbb{R}^{n} \times \mathbb{R}^{n}$ into $\mathbb{R}^{p}$. Moreover, we assume that the pair $(M, m)$ satisfies the classical Kalman rank condition (see [19, Theorem 1.16]). Hence, the state $a$ is fully controllable. We consider the small time null controllability problem for system (1.10). We want to know, if, for any $T>0$, for any initial state $\left(a^{0}, b^{0}\right)$, there exists a control $u:(0, T) \rightarrow \mathbb{R}$ such that the solution to (1.10) satisfies $a(T)=0$ and $b(T)=0$. As proved in [11] for the case $L=0$, the answer to this question is always no in finite dimension, whatever $M, m, L$ and $Q$.

System (1.10) is a particular case of the more general class of control affine systems. Indeed, if we let $x(t)=(a(t), b(t)) \in \mathbb{R}^{n+p}$, we can write system (1.10) as:

$$
\dot{x}=f_{0}(x)+u(t) f_{1}(x),
$$

where $f_{0}(x)=(M a, L b+Q(a, a))$ and $f_{1}(x)=(m, 0)$. The controllability of systems like (1.11) is deeply linked to the iterated Lie brackets of the vector fields $f_{0}$ and $f_{1}$ (see [19, Section 3.2] for a review).

Let us give a few examples with $n=3$. We write $a=\left(a_{1}, a_{2}, a_{3}\right)$ and we consider the system:

$$
\dot{a}_{1}=a_{2}, \quad \dot{a}_{2}=a_{3}, \quad \dot{a}_{3}=u .
$$

Although the strong structure of equation (1.12) can seem a little artificial, it is in fact the general case. Indeed, up to a translation of the control, controllable systems can always be brought back 
to this canonical form introduced by Brunovsky in [12] (for a proof, see [45, Theorem 2.2.7]). The resulting system is flat. We can express the full state as derivatives of a single scalar function. Indeed, if we let $\theta=a_{1}$, we have $a_{2}=\theta^{\prime}, a_{3}=\theta^{\prime \prime}$ and $u=\theta^{\prime \prime \prime}$. If we choose an initial state $\left(a^{0}, b^{0}\right)$ with $a^{0}=0$, we obtain $\theta(0)=\theta^{\prime}(0)=\theta^{\prime \prime}(0)=0$. Moreover, if we assume that the control $u$ drives the state $(a, b)$ to $(0,0)$ at time $T$, we also have $\theta(T)=\theta^{\prime}(T)=\theta^{\prime \prime}(T)=0$. These conditions allow integration by parts without boundary terms.

To keep the examples simple, we choose $p=1$ (hence $b=b_{1} \in \mathbb{R}$ ) and we let $L=0$.

First example. We consider the evolution $\dot{b}=a_{2}^{2}+a_{1} a_{3}$. If the initial state is $\left(a^{0}, b^{0}\right)$ where $a^{0}=0$, we can compute $b(T)=b^{0}+\int_{0}^{T} \theta^{\prime 2}(t)+\theta(t) \theta^{\prime \prime}(t) \mathrm{d} t=b^{0}$. Hence, null controllability does not hold since any control driving $a$ from 0 back to 0 has no action on $b$. This obstruction to controllability is linked to the fact that $\operatorname{dim} \mathcal{L}(0)=3$, where $\mathcal{L}$ is the Lie algebra generated by $f_{0}$ and $f_{1}$. The system is locally constrained to evolve within a 3 dimensional manifold of $\mathbb{R}^{4}$. Indeed, the evolution equation can be rephrased as $\dot{b}=\frac{\mathrm{d}}{\mathrm{d} t}\left(a_{1} a_{2}\right)$. Thus, the quantity $b-a_{1} a_{2}$ is a constant (conservation law of the system).

Second example. We consider the evolution $\dot{b}=a_{3}^{2}$. Thus, $b(T)=b^{0}+\int_{0}^{T} \theta^{\prime \prime}(t)^{2} \mathrm{~d} t$. This is also an obstruction to null controllability. Indeed, all choices of control will make $b$ increase. In this setting, we recover the well known second order Lie bracket condition discovered by Sussmann (see [43, Proposition 6.3]). Indeed, here, $\left[f_{1},\left[f_{1}, f_{0}\right]\right]=\left(0_{\mathbb{R}^{3}}, Q(m, m)\right)=\left(0_{\mathbb{R}^{3}}, 1\right)$. System (1.11) drifts in the direction $\left[f_{1},\left[f_{1}, f_{0}\right]\right]$ and the control cannot prevent it because this direction does not belong to the set of the first order controllable directions $(m, 0),(M m, 0)$ and $\left(M^{2} m, 0\right)$ (Lie brackets of $f_{0}$ and $f_{1}$ involving $f_{1}$ once and only once).

Third example. We consider $\dot{b}=a_{2}^{2}$. Thus, $b(T)=b^{0}+\int_{0}^{T} \theta^{\prime 2}(t) \mathrm{d} t$. Again, $b$ can only increase. Here, the first $b a d$ Lie bracket $\left[f_{1},\left[f_{1}, f_{0}\right]\right]$ vanishes for $x=0$. However, we can check that $\left[f_{1},\left[f_{0},\left[f_{0},\left[f_{1}, f_{0}\right]\right]\right]\right]=\left(0_{\mathbb{R}^{3}}, Q(M m, M m)\right)=\left(0_{\mathbb{R}^{3}}, 1\right)$. Compared with the second example, the increase of $b$ is weaker. Indeed, in the second example, we had $b(T)=b^{0}+|u|_{H^{-1}(0, T)}^{2}$. In this third example, $b(T)=b^{0}+|u|_{H^{-2}(0, T)}^{2}$.

Although these examples may seem caricatural, they reflect the general case. In finite dimension, systems like (1.10) are never small time controllable. Either because they evolve within a strict manifold, or because some quantity depending on $b$ increases. Moreover, the amount by which $b$ increases is linked to the order of the first bad Lie bracket and can be expressed as a weak norm depending on the control. One of the goals of our work is thus also to investigate the situation in infinite dimension, where Lie brackets are harder to define and compute.

Therefore, the first natural question is to compute the Lie bracket $\left[f_{1},\left[f_{1}, f_{0}\right]\right](0)$ for systems $(1.8)$ and (1.9). As we have seen in finite dimension, this Lie bracket is $(0, Q(m, m))$. In our setting, $m$ is the even function $\chi_{[0,1]}$ and $Q(a, a)=-a a_{x}$. Thus $Q(m, m)$ is null. This can be proved computationally using Fourier series expansions. Let us give a much simpler argument inspired by the formal fact that $\partial_{x} 1=0$. For any $a \in L^{2}(0,1)$ and any smooth test function $\phi$ such that $\phi(0)=\phi(1)=0$, we have:

$$
\int_{0}^{1} Q(a, a) \phi=\frac{1}{2} \int_{0}^{1} a^{2}(x) \phi_{x}(x) \mathrm{d} x .
$$

Hence, even if $q:=Q(1,1)$ was defined in a very weak sense, (1.13) yields:

$$
\langle q, \phi\rangle=\frac{1}{2} \int_{0}^{1} \phi_{x}=\frac{1}{2} \phi(1)-\frac{1}{2} \phi(0)=0
$$

Since (1.14) is valid for any smooth $\phi$ null at the boundaries, we conclude that indeed, $q=Q(1,1)$ is null. Therefore, the classical $\left[f_{1},\left[f_{1}, f_{0}\right]\right]$ necessary condition by Sussmann does not provide an obstruction to small time controllability for our system. This also explains why the coercivity property we are going to prove is in a weaker norm than $H^{-1}$.

\subsection{Strategy for the proof}

Most of this paper is dedicated to the asymptotic study of systems (1.8) and (1.9) as the viscosity $\varepsilon$ tends to zero. In Section 6, we prove that this study is sufficient to conclude about the local null controllability for system (1.1). In order to prove that system (1.1) is not small time locally null 
controllable, we intend to exhibit a quantity depending on the state $y(t, \cdot)$ that cannot be controlled. For $\rho \in H^{1}(0,1)$, we will consider quantities of the form $\langle\rho, y(t, \cdot)\rangle$.

Looking at system (1.9) when $\varepsilon$ is very small, we get the idea to consider $\rho(x)=x-\frac{1}{2}$. Indeed, we obtain:

$$
\frac{\mathrm{d}}{\mathrm{d} t} \int_{0}^{1} \rho(x) b(t, x) \mathrm{d} x=\frac{1}{2} \int_{0}^{1} a^{2}(t, x) \mathrm{d} x+\frac{\varepsilon}{2}\left(b_{x}(t, 1)-b_{x}(t, 0)\right) .
$$

Formally, if we let $\varepsilon=0$ in equation (1.15), it is very encouraging because it shows that the quantity $\langle\rho, b\rangle$ can only increase, whatever is the choice of the control. Moreover, since we can compute the amount by which it increases, we have a kind of coercivity and we can hope to be able to use it to overwhelm both residues coming from the fact that $\varepsilon>0$ and residues between the quadratic approximation and the full non-linear system. Sadly, the second term in the right-hand side of equation (1.15) is hard to handle. However, as $a$ depends linearly on $u$, and $b$ depends quadratically on $a$, we expect that we can find a kernel $K^{\varepsilon}\left(s_{1}, s_{2}\right)$ such that:

$$
\langle\rho, b(1, \cdot)\rangle=\int_{0}^{1} \int_{0}^{1} K^{\varepsilon}\left(s_{1}, s_{2}\right) u\left(s_{1}\right) u\left(s_{2}\right) \mathrm{d} s_{1} \mathrm{~d} s_{2} .
$$

Thanks to equation (1.15), we expect that (1.16) actually defines a positive definite kernel acting on $u$, allowing us to use its coercivity to overwhelm various residues.

In Section 2, we recall a set of technical well-posedness estimates for heat and Burgers systems.

In Section 3, we show that formula (1.16) holds and we give an explicit construction of the kernel $K^{\varepsilon}$. Moreover, we compute formally its limit $K^{0}$ as $\varepsilon \rightarrow 0$.

In Section 4, we prove that the kernel $K^{0}$ is coercive with respect to the $H^{-5 / 4}(0,1)$ norm of the control $u$, by recognizing a Riesz potential and a fractional laplacian.

In Section 5 , we use weakly singular integral operator estimates to bound the residues between $K^{\varepsilon}$ and $K^{0}$ and thus deduce that $K^{\varepsilon}$ is also coercive, for $\varepsilon$ small enough.

In Section 6, we use these results to go back to the controllability of Burgers.

In Appendix A, we give a short presentation of the theory of weakly singular integral operators and a sketch of proof of the main estimation lemma we use.

\section{Preliminary technical lemmas}

In this section, we recall a few useful lemmas and estimates, mostly concerning the heat equation and Burgers equation on a line segment. Throughout this section, $\nu$ is a positive viscosity and $T$ a positive time. To lighten the computations, we will use the notation $\lesssim$ to denote inequalities that hold up to a numerical constant. We will not attempt to keep track of these numerical constants. We insist on the fact that these constants do not depend on any parameter (neither the time $T$, nor the viscosity $\nu$, the control $u$, or any other unknown).

\subsection{Properties of the space $X_{T}$}

We recall the definition given in the introduction and state without proof the following classical lemmas which can be proved using either interpolation theory or Fourier transforms with respect to time and space.

Definition 2. We define the functional space:

$$
X_{T}=L^{2}\left((0, T), H^{2}(0,1)\right) \cap H^{1}\left((0, T), L^{2}(0,1)\right) .
$$

We endow the space $X_{T}$ with the scaling invariant norm:

$$
\|z\|_{X_{T}}:=T^{-1 / 2}\|z\|_{2}+T^{-1 / 2}\left\|z_{x x}\right\|_{2}+T^{1 / 2}\left\|z_{t}\right\|_{2} .
$$

Lemma 1. $X_{T} \hookrightarrow \mathcal{C}^{0}\left([0, T], H^{1}(0,1)\right)$. Moreover, for any function $z \in X_{T}$,

$$
\sup _{t \in[0, T]}|z(t, \cdot)|_{H^{1}(0,1)} \lesssim\|z\|_{X_{T}} .
$$

In particular,

$$
\|z\|_{\infty} \lesssim\|z\|_{X_{T}}
$$


Lemma 2. For any $z \in X_{T}$, the boundary traces of $z_{x}$ satisfy:

$$
T^{-1 / 4}\left|z_{x}(\cdot, 0)\right|_{H^{1 / 4}(0, T)}+T^{-1 / 4}\left|z_{x}(\cdot, 1)\right|_{H^{1 / 4}(0, T)} \lesssim\|z\|_{X_{T}} .
$$

\subsection{Smooth setting for the heat equation}

We start by recalling standard estimates in a smooth (strong) setting for one dimensional heat equations that will be useful in the sequel. We state all results for standard forward heat equations, but the same results hold for backwards heat equations with final time conditions.

Lemma 3. Let $f \in L^{2}((0, T) \times(0,1))$ and $z^{0} \in H_{0}^{1}(0,1)$. We consider the system:

$$
\left\{\begin{aligned}
z_{t}-\nu z_{x x} & =f & & \text { in }(0, T) \times(0,1), \\
z(t, 0) & =0 & & \text { in }(0, T), \\
z(t, 1) & =0 & & \text { in }(0, T), \\
z(0, x) & =z^{0}(x) & & \text { in }(0,1) .
\end{aligned}\right.
$$

There is a unique solution $z \in X_{T}$ to system (2.6). Moreover, it satisfies the estimate:

$$
\nu\left\|z_{x x}\right\|_{2}+\sqrt{\nu}\left\|z_{x}\right\|_{2}+\left\|z_{t}\right\|_{2} \lesssim\|f\|_{2}+\sqrt{\nu}\left|z_{x}^{0}\right|_{2} .
$$

Proof. The proof of the existence and uniqueness is standard. Let us recall how we can obtain estimate (2.7). We multiply equation (2.6) by $z_{x x}$ and integrate by parts over $x \in(0,1)$. Thus,

$$
\frac{\mathrm{d}}{\mathrm{d} t}\left[\frac{1}{2} \int_{0}^{1} z_{x}^{2}\right]+\nu \int_{0}^{1} z_{x x}^{2}=-\int_{0}^{1} f z_{x x}
$$

For any $T^{\prime}<T$, we can integrate $(2.8)$ over $t \in\left(0, T^{\prime}\right)$. Hence, we obtain:

$$
\frac{1}{2}\left|z_{x}\left(T^{\prime}\right)\right|_{2}^{2}+\nu \int_{0}^{T^{\prime}} \int_{0}^{1} z_{x x}^{2}=-\int_{0}^{T^{\prime}} \int_{0}^{1} f z_{x x}+\frac{1}{2}\left|z_{x}^{0}\right|_{2}^{2} .
$$

From (2.9), we easily deduce that:

$$
\begin{aligned}
\nu\left\|z_{x x}\right\|_{2} & \lesssim\|f\|_{L^{2}}+\sqrt{\nu}\left|z_{x}^{0}\right|_{2}, \\
\sqrt{\nu}\left\|z_{x}\right\|_{L^{\infty}\left(L^{2}\right)} & \lesssim\|f\|_{L^{2}}+\sqrt{\nu}\left|z_{x}^{0}\right|_{2} .
\end{aligned}
$$

Eventually, we obtain estimate (2.7) from estimates (2.10) and (2.11) since we can write $z_{t}$ as $f+$ $\nu z_{x x}$.

Lemma 4. Let $z^{0} \in H_{0}^{1}(0,1)$ and consider $z \in X_{T}$ the solution to system (2.6) with a null forcing term $(f=0)$. It satisfies:

$$
\|z\|_{\infty} \leq\left|z^{0}\right|_{\infty}
$$

Proof. Although (2.12) is not a direct consequence of the combination of (2.4) and (2.7) (which would yield a weaker conclusion), it can be obtained via a standard application of the maximum principle, which can be applied in this strong setting.

\subsection{Weaker settings for the heat equation}

Let us move on to weaker settings for the heat equation. Moreover, we introduce inhomogeneous boundary data as we will need them in the sequel.

Definition 3. Let $f \in\left(X_{T}\right)^{\prime}, v_{0}, v_{1} \in H^{-1 / 4}(0, T)$ and $z^{0} \in H^{-1}(0,1)$. We consider:

$$
\left\{\begin{aligned}
z_{t}-\nu z_{x x} & =f & & \text { in }(0, T) \times(0,1), \\
z(t, 0) & =v_{0}(t) & & \text { in }(0, T), \\
z(t, 1) & =v_{1}(t) & & \text { in }(0, T), \\
z(0, x) & =z^{0}(x) & & \text { in }(0,1) .
\end{aligned}\right.
$$


We say that $z \in L^{2}((0, T) \times(0,1))$ is a weak solution to system $(2.13)$ if, for all $g \in L^{2}((0, T) \times(0,1))$,

$$
\begin{aligned}
\langle z, g\rangle_{L^{2}, L^{2}}=\langle f, \varphi\rangle_{\left(X_{T}\right)^{\prime}, X_{T}} & +\left\langle z^{0}, \varphi(0, \cdot)\right\rangle_{H^{-1}(0,1), H_{0}^{1}(0,1)} \\
& +\nu\left\langle v_{0}, \varphi_{x}(\cdot, 0)\right\rangle_{H^{-1 / 4}(0, T), H^{1 / 4}(0, T)} \\
& -\nu\left\langle v_{1}, \varphi_{x}(\cdot, 1)\right\rangle_{H^{-1 / 4}(0, T), H^{1 / 4}(0, T)}
\end{aligned}
$$

where $\varphi \in X_{T}$ is the solution to the dual system:

$$
\left\{\begin{aligned}
\varphi_{t}+\nu \varphi_{x x} & =-g & & \text { in }(0, T) \times(0,1) \\
\varphi(t, 0) & =0 & & \text { in }(0, T) \\
\varphi(t, 1) & =0 & & \text { in }(0, T) \\
\varphi(T, x) & =0 & & \text { in }(0,1)
\end{aligned}\right.
$$

Lemma 5. There exists a unique weak solution $z \in L^{2}((0, T) \times(0,1))$ to system (2.13). Moreover:

$$
\|z\|_{2} \lesssim T^{-1 / 2} \nu^{-1}\left(\|f\|_{\left(X_{T}\right)^{\prime}}+\left|z^{0}\right|_{H^{-1}}\right)+T^{-1 / 4}\left(\left|v_{0}\right|_{H^{-1 / 4}}+\left|v_{1}\right|_{H^{-1 / 4}}\right) .
$$

Proof. For any $g \in L^{2}((0, T) \times(0,1))$, Lemma 3 asserts that system $(2.15)$ admits a unique solution $\varphi \in X_{T}$ such that $\|\varphi\|_{X_{T}} \lesssim T^{-1 / 2} \nu^{-1}\|g\|_{L^{2}}$. Moreover, thanks to estimates (2.3) and (2.5), the right-hand side of equation (2.14) defines a continuous linear form on $L^{2}$. The Riesz representation theorem therefore proves the existence of a unique $z \in L^{2}$ satisfying estimate (2.16).

Lemma 6. Let $f \in L^{2}((0, T) \times(0,1))$. We consider the following heat system:

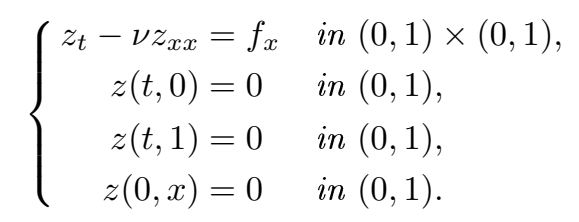

There is a unique solution $z \in L^{2}((0, T) \times(0,1))$ to system $(2.17)$. Moreover, it satisfies the estimate:

$$
\nu^{1 / 2}\|z\|_{L^{\infty}\left(L^{2}\right)}+\nu\left\|z_{x}\right\|_{L^{2}} \lesssim\|f\|_{L^{2}} .
$$

Proof. For $f \in L^{2}$, it is easy to check that $f_{x} \in X_{T}^{\prime}$. Hence, we can apply Lemma 5 and system (2.17) has a unique solution $z \in L^{2}$. In fact, this solution is even smoother. Estimate (2.18) is obtained as usual by multiplying equation (2.17) by $z$ and integration by parts.

\subsection{Burgers and forced Burgers systems}

We move on to Burgers-like systems. For the sake of completeness, we provide a short proof of the existence of a solution to system (1.1) and a precise estimate for forced Burgers-like systems that will be necessary in the sequel.

Lemma 7. Let $w \in X_{T}, g \in L^{2}\left((0, T), H^{1}(0,1)\right)$ and $y^{0} \in H_{0}^{1}(0,1)$. We consider $y \in X_{T}$ a solution to the following forced Burgers-like system:

$$
\left\{\begin{aligned}
y_{t}-\nu y_{x x} & =-y y_{x}+(w y)_{x}+g_{x} & & \text { in }(0, T) \times(0,1), \\
y(t, 0) & =0 & & \text { in }(0, T), \\
y(t, 1) & =0 & & \text { in }(0, T), \\
y(0, x) & =y^{0}(x) & & \text { in }(0,1) .
\end{aligned}\right.
$$

Then,

$$
\begin{aligned}
\nu\left\|y_{x x}\right\|_{2}+\sqrt{\nu}\left\|y_{x}\right\|_{2}+\left\|y_{t}\right\|_{2} \lesssim & \left\|g_{x}\right\|_{2}+e^{\gamma}\left\|w_{x}\right\|_{L^{2}\left(L^{\infty}\right)}\left(\nu^{-1 / 2}\|g\|_{2}+\left|y^{0}\right|_{2}^{2}\right) \\
& +\left(1+\sqrt{\gamma} e^{\gamma}\right)\|w\|_{\infty}\left(\nu^{-1}\|g\|_{2}+\nu^{-1 / 2}\left|y^{0}\right|_{2}^{2}\right) \\
& +\left(1+\sqrt{\gamma} e^{6 \gamma}\right) e^{\gamma}\|g\|_{L^{2}\left(L^{\infty}\right)}\left(\nu^{-3 / 2}\|g\|_{2}+\nu^{-1}\left|y^{0}\right|_{2}\right) \\
& +\left(1+\sqrt{\gamma} e^{6 \gamma}\right) \nu^{-1 / 2}\left|y^{0}\right|_{4}^{2}+\nu^{1 / 2}\left|y_{x}^{0}\right|_{2} .
\end{aligned}
$$

where we introduce $\gamma=\frac{1}{\nu}\|w\|_{L^{2}\left(L^{\infty}\right)}^{2}$. 
Proof. $L^{2}$ estimates for $y$ and $y_{x}$. We start by multiplying equation (2.19) by $y$, and integrate by parts over $(0,1)$ :

$$
\begin{aligned}
\frac{1}{2} \frac{\mathrm{d}}{\mathrm{d} t} \int_{0}^{1} y^{2}+\nu \int_{0}^{1} y_{x}^{2} & =-\int_{0}^{1} w y y_{x}-\int_{0}^{1} g y_{x} \\
& \leq \frac{2}{2 \nu} \int_{0}^{1} w^{2} y^{2}+\frac{\nu}{4} \int_{0}^{1} y_{x}^{2}+\frac{2}{2 \nu} \int_{0}^{1} g^{2}+\frac{\nu}{4} \int_{0}^{1} y_{x}^{2} .
\end{aligned}
$$

From (2.21), we deduce:

$$
\frac{\mathrm{d}}{\mathrm{d} t} \int_{0}^{1} y^{2}+\nu \int_{0}^{1} y_{x}^{2} \leq \frac{2}{\nu}|w(t, \cdot)|_{\infty}^{2} \int_{0}^{1} y^{2}+\frac{2}{\nu} \int_{0}^{1} g^{2} .
$$

We apply Grönwall's lemma to (2.22) to obtain:

$$
\|y\|_{L^{\infty}\left(L^{2}\right)}^{2} \leq e^{2 \gamma}\left(\frac{2}{\nu}\|g\|_{2}^{2}+\left|y^{0}\right|_{2}^{2}\right) .
$$

Plugging (2.23) into (2.22) yields:

$$
\nu\left\|y_{x}\right\|_{2}^{2} \leq\left(1+2 \gamma e^{2 \gamma}\right)\left(\frac{2}{\nu}\|g\|_{2}^{2}+\left|y^{0}\right|_{2}^{2}\right) .
$$

$L^{2}$ estimate for $y y_{x}$. We repeat a similar technique, multiplying this time equation (2.19) by $y^{3}$. Using the same approach yields:

$$
\frac{\mathrm{d}}{\mathrm{d} t} \int_{0}^{1} y^{4}+6 \nu \int_{0}^{1} y^{2} y_{x}^{2} \leq \frac{12}{\nu}|w(t, \cdot)|_{\infty}^{2} \int_{0}^{1} y^{4}+\frac{12}{\nu}|g(t, \cdot)|_{\infty}^{2} \int_{0}^{1} y^{2} .
$$

We apply Grönwall's lemma to (2.25) to obtain:

$$
\|y\|_{L^{\infty}\left(L^{4}\right)}^{4} \leq e^{12 \gamma}\left(\frac{12}{\nu}\|g\|_{L^{2}\left(L^{\infty}\right)}^{2}\|y\|_{L^{\infty}\left(L^{2}\right)}^{2}+\left|y^{0}\right|_{4}^{4}\right) .
$$

Once again, plugging back estimate (2.26) into (2.25) gives:

$$
6 \nu\left\|y y_{x}\right\|_{2}^{2} \leq\left(1+12 \gamma e^{12 \gamma}\right)\left(\frac{12}{\nu}\|g\|_{L^{2}\left(L^{\infty}\right)}^{2}\|y\|_{L^{\infty}\left(L^{2}\right)}^{2}+\left|y^{0}\right|_{4}^{4}\right) .
$$

Conclusion. To conclude the proof, we use Lemma 3, with a source term $f=g_{x}+w_{x} y+w y_{x}-y y_{x}$. Estimate (2.20) comes from the combination of (2.7) with equations (2.23), (2.24) and (2.27).

Lemma 8. For any initial data $y_{0} \in H_{0}^{1}(0,1)$ and any control $u \in L^{2}(0, T)$, system (1.1) has a unique solution $y \in X_{T}$. Moreover:

$$
\begin{aligned}
\left\|y_{x x}\right\|_{2}+\left\|y_{t}\right\|_{2} & \lesssim|u|_{2}+|u|_{2}^{2}+\left|y^{0}\right|_{4}^{2}+\left|y_{x}^{0}\right|_{2}, \\
\|y\|_{\infty} & \leq\left|y^{0}\right|_{\infty}+|u|_{L^{1}} .
\end{aligned}
$$

Proof. This type of existence result relies on standard a priori estimates and the use of a fixed point theorem. Such techniques are described in [34]. One can also use a semi-group method as in [38]. The quantitative estimate is obtained by applying Lemma 7 with $w=0$ (hence $\gamma=0$ ) and $g(t, x)=x u(t)$. Equation (2.20) yields (2.28). The second estimate (2.29) is a consequence of the maximum principle, which can be applied in this strong setting.

\section{From Burgers to a kernel integral operator}

\subsection{A general method for evaluating a projection}

As we mentionned in the introduction, we are going to consider a projection of the state $b$ against some given profile $\rho(x)$ at the final time $t=1$. Since $a$ depends linearly on $u$ and $b$ depends quadratically on $a$, it is natural to look for this projection as a quadratic integral operator acting on our control $u$. Indeed, let us prove the following result. 
Lemma 9. Let $\rho \in L^{2}(0,1)$ and $\varepsilon>0$. There exists a symmetric kernel $K^{\varepsilon} \in L^{\infty}\left((0,1)^{2}\right)$ such that, for any $u \in L^{2}(0,1)$, the solution to system (1.8)-(1.9) satisfies:

$$
\int_{0}^{1} b(1, x) \rho(x) \mathrm{d} x=\iint_{(0,1)^{2}} K^{\varepsilon}\left(s_{1}, s_{2}\right) u\left(s_{1}\right) u\left(s_{2}\right) \mathrm{d} s_{1} \mathrm{~d} s_{2} .
$$

The key point of the proof is to convert the pointwise in time projection of $b$ into an integrated projection over the time interval $(0,1)$. Indeed, we start with the proof of the following lemma.

Lemma 10. Let $f \in L^{2}\left((0,1)^{2}\right), \varepsilon>0$ and $z \in X_{1}$ be the solution to:

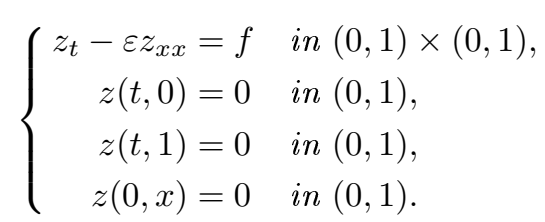

Take $\rho \in L^{2}(0,1)$. The final time projection of $z$ against $\rho$ satisfies:

$$
\int_{0}^{1} z(1, x) \rho(x) \mathrm{d} x=\iint_{(0,1)^{2}} \Phi(1-t, x) f(t, x) \mathrm{d} x \mathrm{~d} t
$$

where $\Phi \in X_{1}$ is the solution to:

$$
\left\{\begin{aligned}
\Phi_{t}-\varepsilon \Phi_{x x} & =0 & & \text { in }(0,1) \times(0,1), \\
\Phi(t, 0) & =0 & & \text { in }(0,1), \\
\Phi(t, 1) & =0 & & \text { in }(0,1), \\
\Phi(0, x) & =\rho(x) & & \text { in }(0,1) .
\end{aligned}\right.
$$

Proof. Let us introduce $\Psi \in X_{1}$, the solution to:

$$
\left\{\begin{aligned}
\Psi_{t}-\varepsilon \Psi_{x x}=\rho & \text { in }(0,1) \times(0,1), \\
\Psi(t, 0)=0 & \text { in }(0,1), \\
\Psi(t, 1)=0 & \text { in }(0,1), \\
\Psi(0, x)=0 & \text { in }(0,1) .
\end{aligned}\right.
$$

Using this system, we can convert the time punctual projection of the state $z$ against $\rho$ into a projection of the source term $f$ onto the full square:

$$
\begin{aligned}
\int_{0}^{1} z(1, x) \rho(x) \mathrm{d} x & =\left.\frac{\mathrm{d}}{\mathrm{d} T} \int_{0}^{T} \int_{0}^{1} z(t, x) \cdot \rho(x) \mathrm{d} x \mathrm{~d} t\right|_{T=1} \\
& =\left.\frac{\mathrm{d}}{\mathrm{d} T} \int_{0}^{T} \int_{0}^{1} z(t, x) \cdot\left\{\Psi_{t}-\varepsilon \Psi_{x x}\right\}(T-t, x) \mathrm{d} x \mathrm{~d} t\right|_{T=1} \\
& =\left.\frac{\mathrm{d}}{\mathrm{d} T} \int_{0}^{T} \int_{0}^{1}\left\{z_{t}-\varepsilon z_{x x}\right\}(t, x) \cdot \Psi(T-t, x) \mathrm{d} x \mathrm{~d} t\right|_{T=1} \\
& =\left.\frac{\mathrm{d}}{\mathrm{d} T} \int_{0}^{T} \int_{0}^{1} f(t, x) \cdot \Psi(T-t, x) \mathrm{d} x \mathrm{~d} t\right|_{T=1} \\
& =\int_{0}^{1} \int_{0}^{1} f(t, x) \Psi_{t}(1-t, x) \mathrm{d} x \mathrm{~d} t
\end{aligned}
$$

The integrations by parts performed above are valid because of the null boundary and initial conditions chosen in systems (3.2) and (3.5). Equation (3.3) is a direct consequence of (3.6) since $\Psi_{t}=\Phi$.

Let us come back to the proof of Lemma 9. We apply Lemma 10 to the state $b$. Thus, from (1.9) and (3.3) we deduce that:

$$
\begin{aligned}
\int_{0}^{1} b(1, x) \rho(x) \mathrm{d} x & =\int_{0}^{1} \int_{0}^{1} \Phi(1-t, x)\left[-a a_{x}\right](t, x) \mathrm{d} x \mathrm{~d} t \\
& =\frac{1}{2} \int_{0}^{1} \int_{0}^{1} \Phi_{x}(1-t, x) a^{2}(t, x) \mathrm{d} x \mathrm{~d} t
\end{aligned}
$$


In order to express our projection directly using $u$, we need to eliminate $a$ from (3.7). This can easily be done using an elementary solution of the heat system. Therefore, we introduce $G$ the solution to:

$$
\left\{\begin{aligned}
G_{t}-\varepsilon G_{x x}=0 & \text { in }(0,1) \times(0,1), \\
G(t, 0)=0 & \text { in }(0,1), \\
G(t, 1)=0 & \text { in }(0,1), \\
G(0, x)=1 & \text { in }(0,1) .
\end{aligned}\right.
$$

Using the initial condition $a(t=0, \cdot) \equiv 0$ from system (1.8), we can expand $a$ as:

$$
a(t, x)=\int_{0}^{t} G(t-s, x) u(s) \mathrm{d} s .
$$

Pluging (3.9) into (3.7) yields:

$$
\begin{aligned}
\int_{0}^{1} b(1, x) \rho(x) \mathrm{d} x & =\frac{1}{2} \int_{0}^{1} \int_{0}^{1} \Phi_{x}(1-t)\left(\int_{0}^{t} G\left(t-s_{1}\right) u\left(s_{1}\right) \mathrm{d} s_{1}\right)\left(\int_{0}^{t} G\left(t-s_{2}\right) u\left(s_{2}\right) \mathrm{d} s_{2}\right) \mathrm{d} t \\
& =\frac{1}{2} \int_{0}^{1} \int_{0}^{1} u\left(s_{1}\right) u\left(s_{2}\right)\left(\int_{s_{1} \vee s_{2}}^{1} \int_{0}^{1} \Phi_{x}(1-t) G\left(t-s_{1}\right) G\left(t-s_{2}\right) \mathrm{d} t\right) \mathrm{d} s_{1} \mathrm{~d} s_{2} .
\end{aligned}
$$

Finally, equation (3.10) proves (3.1) with:

$$
K^{\varepsilon}\left(s_{1}, s_{2}\right)=\frac{1}{2} \int_{s_{1} \vee s_{2}}^{1} \int_{0}^{1} \Phi_{x}(1-t, x) G\left(t-s_{1}, x\right) G\left(t-s_{2}, x\right) \mathrm{d} x \mathrm{~d} t .
$$

Thus, we have proved Lemma 9 and we have a very precise description of the kernel that is involved. This kernel depends on the projection profile $\rho(x)$ by means of $\Phi$ defined in (3.4). This kernel also strongly depends on the viscosity $\varepsilon$ which is involded in the computation of both $\Phi$ and of the elementary solution $G$.

Moreover, it is clear that $K$ is a symmetric kernel and since all terms are bounded thanks to the maximum principle, we know that $K \in L^{\infty}$. In fact, $K$ is even smoother as we will see later on.

\subsection{Choice of a profile $\rho$}

As we have seen in the introduction, a natural choice in the low viscosity setting would be $\rho(x)=x-\frac{1}{2}$. We think that our proof could be adapted to work with this profile. However, the computations are tough because it does not satisfy null boundary conditions. Thus, we are going to make a choice which is more intrinsic to the Burgers system.

For any fixed control value $\bar{u} \in \mathbb{R}$, we want to compute the associated steady state $(\bar{a}(x), \bar{b}(x))$ of systems (1.8) and (1.9). Thus, we solve the following system:

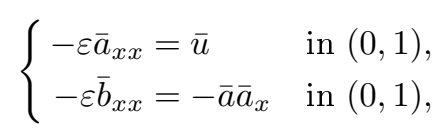

with boundary conditions $\bar{a}(0)=\bar{a}(1)=\bar{b}(0)=\bar{b}(1)=0$. Integrating (3.12) with respect to $x$ yields the following family of steady states:

$$
\bar{a}(x)=\frac{1}{2 \varepsilon} x(1-x) \bar{u} \quad \text { and } \quad \bar{b}(x)=\frac{1}{8 \varepsilon^{3}}\left(\frac{x^{5}}{5}-\frac{x^{4}}{2}+\frac{x^{3}}{3}-\frac{x}{30}\right) \bar{u}^{2} .
$$

Of course, $\bar{b}$ depends quadratically on $\bar{u}$. Thus equation (3.13) gives the idea of considering:

$$
\rho(x)=\frac{x^{5}}{5}-\frac{x^{4}}{2}+\frac{x^{3}}{3}-\frac{x}{30} .
$$

This choice of $\rho$ may seem strange because is has been obtained using an infinite viscosity limit. However, since both $\rho$ and $\rho_{x x}$ satisfy null boundary conditions, the computations of the different kernel residues turn out to be easier. In the sequel, we assume that $\rho$ is defined by (3.14). 


\subsection{Rough computation of the asymptotic kernel}

In this paragraph, we apply Lemma 9 to compute the kernel associated to the choice of $\rho$ given in (3.14). More specifically, we are interested in computing a rough approximation of $K^{\varepsilon}$ when $\varepsilon \rightarrow 0$. This approximation will serve as a motivation for the following sections where we will need to estimate all the residues that will be leaving aside for the moment. Since formula (3.11) defining $K^{\varepsilon}$ involves both $\Phi$ and $G$, we need to choose approximations of these quantities as $\varepsilon \rightarrow 0$. Looking at system (3.4) defining $\Phi$, we choose to use:

$$
\Phi_{x}(t, x) \approx \rho_{x}(x) .
$$

Moreover, for $G$ defined by (3.8), we will use the approximation $G \approx 1$ inside $(0,1)$. Stopping here would not yield anything useful. Indeed, since $\int_{0}^{1} \rho_{x}=\rho(1)-\rho(0)=0$, we would obtain $K^{\varepsilon}=0$. Hence, we need to choose an approximation of $G$ that is more accurate near the boundary, eg:

$$
G(t, x) \approx \operatorname{erf}\left(\frac{x}{\sqrt{4 \varepsilon t}}\right),
$$

which we will use near $x=0$. Note that equation (3.16) corresponds to the solution of a heat equation on the real line with an initial data equal to -1 for $x<0$ and +1 for $x>0$. Thus, it satisfies the boundary condition $G(t, 0) \equiv 0$ and serves as a boundary layer correction. We compute the integrand inside equation (3.11):

$$
\begin{array}{rlrl}
A^{\varepsilon}\left(t, s_{1}, s_{2}\right) & :=\frac{1}{2} \int_{0}^{1} \Phi_{x}(1-t, x) G\left(t-s_{1}, x\right) G\left(t-s_{2}, x\right) \mathrm{d} x & \\
& =\frac{1}{2} \int_{0}^{1} \Phi_{x}(1-t, x)\left(G\left(t-s_{1}, x\right) G\left(t-s_{2}, x\right)-1\right) \mathrm{d} x & \text { since } \int \Phi_{x}=0 \\
& =\int_{0}^{\frac{1}{2}} \Phi_{x}(1-t, x)\left(G\left(t-s_{1}, x\right) G\left(t-s_{2}, x\right)-1\right) \mathrm{d} x & \text { by parity }, \\
& \approx \int_{0}^{\frac{1}{2}} \rho_{x}(x)\left(\operatorname{erf}\left(\frac{x}{\sqrt{4 \varepsilon\left(t-s_{1}\right)}}\right) \operatorname{erf}\left(\frac{x}{\sqrt{4 \varepsilon\left(t-s_{2}\right)}}\right)-1\right) \mathrm{d} x & \text { using }(3.15),(3.16), \\
& \approx 2 \sqrt{\varepsilon} \int_{0}^{\frac{1}{4 \sqrt{\varepsilon}}} \rho_{x}(2 \sqrt{\varepsilon} x)\left(\operatorname{erf}\left(\frac{x}{\sqrt{\left(t-s_{1}\right)}}\right) \operatorname{erf}\left(\frac{x}{\sqrt{\left(t-s_{2}\right)}}\right)-1\right) \mathrm{d} x & \\
& \sim-2 \sqrt{\varepsilon} \rho_{x}(0) \int_{0}^{+\infty}\left(1-\operatorname{erf}\left(\frac{x}{\sqrt{\left(t-s_{1}\right)}}\right) \operatorname{erf}\left(\frac{x}{\sqrt{\left(t-s_{2}\right)}}\right)\right) \mathrm{d} x .
\end{array}
$$

To carry on with the computation, we need the following integral calculus lemma.

Lemma 11. Let $\alpha, \beta>0$. Then,

$$
\int_{0}^{+\infty}(1-\operatorname{erf}(\alpha x) \operatorname{erf}(\beta x)) \mathrm{d} x=\frac{1}{\alpha \beta} \sqrt{\frac{\alpha^{2}+\beta^{2}}{\pi}} .
$$

Proof. We can find an explicit primitive for the integrand. Indeed, for any $X>0$,

$$
\begin{aligned}
\int_{0}^{X}(1-\operatorname{erf}(\alpha x) \operatorname{erf}(\beta x)) \mathrm{d} x= & X(1-\operatorname{erf}(\alpha X) \operatorname{erf}(\beta X)) \\
& -\frac{\operatorname{erf}(\alpha X) \exp \left(-\beta^{2} X^{2}\right)}{\beta \sqrt{\pi}}-\frac{\operatorname{erf}(\beta X) \exp \left(-\alpha^{2} X^{2}\right)}{\alpha \sqrt{\pi}} \\
& +\frac{\sqrt{\alpha^{2}+\beta^{2}}}{\alpha \beta \sqrt{\pi}} \operatorname{erf}\left(\sqrt{\alpha^{2}+\beta^{2}} X\right) .
\end{aligned}
$$

Equation (3.19) can be checked by differentiation. Taking its limit as $X \rightarrow+\infty$ yields (3.18).

We return to the computation of the asymptotic kernel as $\varepsilon \rightarrow 0$. We note that $\rho_{x}(0)=-\frac{1}{30}$. 
Combined with (3.11), (3.17) and Lemma 11, we obtain:

$$
\begin{aligned}
K^{\varepsilon}\left(s_{1}, s_{2}\right) & =\int_{s_{1} \vee s_{2}}^{1} A^{\varepsilon}\left(t, s_{1}, s_{2}\right) \mathrm{d} t \\
& \approx \frac{\sqrt{\varepsilon}}{15 \sqrt{\pi}} \int_{s_{1} \vee s_{2}}^{1} \sqrt{\left(t-s_{1}\right)+\left(t-s_{2}\right)} \mathrm{d} t \\
& \approx \frac{\sqrt{\varepsilon}}{45 \sqrt{\pi}} \cdot\left[\left(2 t-s_{1}-s_{2}\right)^{\frac{3}{2}}\right]_{s_{1} \vee s_{2}}^{1} \\
& \approx \frac{\sqrt{\varepsilon}}{45 \sqrt{\pi}} K^{0}\left(s_{1}, s_{2}\right),
\end{aligned}
$$

where we introduce the asymptotic kernel:

$$
K^{0}\left(s_{1}, s_{2}\right)=\left(2-s_{1}-s_{2}\right)^{3 / 2}-\left|s_{1}-s_{2}\right|^{3 / 2} .
$$

At this stage, equation (3.20) is not rigorous. The meaning of the $\approx$ sign has to be made precise. This is the goal of Section 5 where we prove that this asymptotic formula does make sense. Indeed, we estimate the kernel residues between $K^{\varepsilon}$ and $\sqrt{\varepsilon} K^{0}$. They turn out to be both small (with respect to $\varepsilon$ ) and smooth (with respect to the spaces on which they define continuous quadratic forms).

\section{Coercivity of the asymptotic kernel}

In this section, our goal is to prove the coercivity of the kernel $K^{0}(x, y)$. This is a symmetric realvalued kernel defined on $(0,1) \times(0,1)$. Note that, since no confusion is possible, we will use $(x, y)$ instead of $\left(s_{1}, s_{2}\right)$ for the variables of the kernel to lighten notations of this section. We will prove the following theorem.

Lemma 12. The integral operator associated to $K^{0}$ is coercive in the space $H^{-5 / 4}(0,1)$. There exists $\gamma>0$ such that, for any $f \in L^{2}(0,1)$, the following inequality holds:

$$
\int_{0}^{1} \int_{0}^{1} K^{0}(x, y) f(x) f(y) \mathrm{d} x \mathrm{~d} y \geq \gamma\|F\|_{H^{-1 / 4}(0,1)}^{2},
$$

where $F$ is the primitive of $f$ such that $F(0)=0$.

Thanks to the change of variables $(x, y) \mapsto(1-x, 1-y)$, the kernel $K^{0}$ behaves exactly like:

$$
N(x, y)=(x+y)^{3 / 2}-|x-y|^{3 / 2} .
$$

In this section, we will thus study the properties of $N$ whose expression is easier to handle.

\subsection{The kernel $N$ is positive definite}

This section uses results and notions from [9]. We will say that a matrix $A$ is positive semidefinite (psd) when $\langle A x \mid x\rangle \geq 0$ for any $x \in \mathbb{R}^{m}$. We will say that $A$ is positive definite if the inequality is strict for any $x \neq 0$. We will say that $A$ is conditionnaly negative semidefinite (cnsd) when $\langle A x \mid x\rangle \leq 0$ for any $x$ such that $\sum x_{i}=0$. We will use similar definitions for operators.

Lemma 13. For any $f \in L^{2}(0,1)$,

$$
\int_{0}^{1} \int_{0}^{1} N(x, y) f(x) f(y) \mathrm{d} x \mathrm{~d} y \geq 0 .
$$

Proof. All necessary arguments can be found in [9, Chapter 3]. Indeed, the kernel $-(x+y)^{3 / 2}$ is cnsd. as is proved in [9, Corollary 2.11]. Moreover, the kernel $|x-y|^{3 / 2}$ is also $c n s d$. (see [9, Remark 1.10] and [9, Corollary 2.10]). Hence, letting:

$$
\psi(x, y)=-(x+y)^{3 / 2}+|x-y|^{3 / 2}
$$

defines a $c n s d$. kernel. Thus, since:

$$
N(x, y)=\psi(x, 0)+\psi(y, 0)-\psi(x, y)-\psi(0,0),
$$

this kernel is $p s d$. thanks to [9, Lemma 2.1]. This proves inequality (4.3). 
Even though it is true that the kernels involved in the proof of Lemma 13 are striclty negative (or positive), we cannot adapt the proof to prove that $N$ is definite. Indeed, Mercer's theorem (which allows us to take the step from matrices to continuous kernels) doesn't preserve strict inequalities. Thus, we have to look for another proof.

\subsection{Some insight and facts}

Our main insight is that the kernel $N$ is made up of two parts. The most singular one should explain its behavior. Indeed, kernels which can be expressed as a function $r(|x-y|)$ have been extensively studied. For example, [46] and [40] prove asymptotic formulas for the eigenvalues of the $-|x-y|^{3 / 2}$ part of our kernel:

$$
\lambda_{n} \sim \frac{3 \sqrt{2}}{4 \pi^{2}}\left(\frac{1}{n}\right)^{\frac{5}{2}} .
$$

Moreover, some papers have also studied the eigenvectors of such kernels. For example, in [37], one can find asymptotic developments for eigenvectors of kernels of the form $|x-y|^{-\alpha}$, where $\alpha \in(0,1)$.

Combining the insight that the eigenvectors of $N$ should asymptotically behave like oscillating sinuses and formula (4.6), we expect that it should be possible to prove Lemma 12 by means of such an asymptotic study. However, we have not been able to prove it using this method. Instead, we give below a proof based on Riesz potentials.

\subsection{Highlighting the singular part of $N$}

The kernel $N(x, y)$ is rather smooth. In order to prove its coercivity, we will need to isolate it's most singular part. In the following lemma, we use integration by parts twice to show that studying the behavior of $N$ is equivalent to studying a more singular kernel. By choosing adequatly the primitive, we show that we can also cancel boundary terms.

Lemma 14. Let $f \in L^{2}(0,1)$ and $F$ be the primitive of $f$ such that $F(1)=0$. Then:

$$
(N f, f)=\frac{3}{4} \int_{0}^{1} \int_{0}^{1}\left((x+y)^{-\frac{1}{2}}+|x-y|^{-\frac{1}{2}}\right) F(x) F(y) \mathrm{d} x \mathrm{~d} y .
$$

Proof. Let $f \in L^{2}(0,1)$ and $F$ be the primitive of $f$ such that $F(1)=0$. We start with:

$$
\begin{aligned}
-\int_{0}^{1} \int_{0}^{1}|x-y|^{\frac{3}{2}} f(x) f(y) \mathrm{d} x \mathrm{~d} y \\
=-\int_{0}^{1} f(x)\left\{\int_{0}^{x}(x-y)^{\frac{3}{2}} f(y) \mathrm{d} y+\int_{x}^{1}(y-x)^{\frac{3}{2}} f(y) \mathrm{d} y\right\} \mathrm{d} x \\
=F(0) \int_{0}^{1} x^{\frac{3}{2}} f(x) \mathrm{d} x+\frac{3}{2} \int_{0}^{1} \int_{0}^{1}|x-y|^{\frac{1}{2}} \mathrm{sg}(y-x) f(x) F(y) \mathrm{d} x \mathrm{~d} y \\
=F(0) \int_{0}^{1} x^{\frac{3}{2}} f(x) \mathrm{d} x+\frac{3}{2} \int_{0}^{1} F(y)\left\{\int_{0}^{y}(y-x)^{\frac{1}{2}} f(x) \mathrm{d} x-\int_{y}^{1}(x-y)^{\frac{1}{2}} f(x) \mathrm{d} x\right\} \mathrm{d} y \\
=F(0) \int_{0}^{1}\left(x^{\frac{3}{2}} f(x)-\frac{3}{2} x^{\frac{1}{2}} F(x)\right) \mathrm{d} x+\frac{3}{4} \int_{0}^{1} \int_{0}^{1}|x-y|^{-\frac{1}{2}} F(x) F(y) \mathrm{d} x \mathrm{~d} y .
\end{aligned}
$$

We continue with the other half of the kernel $N(x, y)$ :

$$
\begin{aligned}
\int_{0}^{1} \int_{0}^{1} & (x+y)^{\frac{3}{2}} f(x) f(y) \mathrm{d} x \mathrm{~d} y \\
\quad= & -F(0) \int_{0}^{1} x^{\frac{3}{2}} f(x) \mathrm{d} x-\frac{3}{2} \int_{0}^{1} \int_{0}^{1}(x+y)^{\frac{1}{2}} f(x) F(y) \mathrm{d} x \mathrm{~d} y \\
& =F(0) \int_{0}^{1}\left(\frac{3}{2} x^{\frac{1}{2}} F(x)-x^{\frac{3}{2}} f(x)\right) \mathrm{d} x+\frac{3}{4} \int_{0}^{1} \int_{0}^{1}(x+y)^{-\frac{1}{2}} F(x) F(y) \mathrm{d} x \mathrm{~d} y .
\end{aligned}
$$

Summing the two previous equalities proves Lemma 14. 


\subsection{Riesz potential and fractional laplacian}

In this section, we focus on the most singular part of the kernel. We recognize a Riesz potential of order $\frac{1}{2}$. Using the fractional laplacian, we can compute the quantity as a usual norm.

Lemma 15. There exists $C>0$ such that, for any $h \in L^{2}(0,1)$,

$$
\int_{0}^{1} \int_{0}^{1}|x-y|^{-\frac{1}{2}} h(x) h(y) \mathrm{d} x \mathrm{~d} y \geq C\|h\|_{H^{-1 / 4}(0,1)}^{2} .
$$

Proof.

$$
\begin{aligned}
\int_{0}^{1} \int_{0}^{1}|x-y|^{-\frac{1}{2}} h(x) h(y) \mathrm{d} x \mathrm{~d} y & =\int_{\mathbb{R}} \int_{\mathbb{R}}|x-y|^{-\frac{1}{2}} h(x) h(y) \mathrm{d} x \mathrm{~d} y \\
& =\left((-\Delta)^{-1 / 4} h, h\right) \\
& =\left((-\Delta)^{-1 / 8} h,(-\Delta)^{-1 / 8} h\right) \\
& =\left\|(-\Delta)^{-1 / 8} h\right\|_{L^{2}}^{2} \\
& =\|h\|_{\dot{H}^{-1 / 4}}^{2} \\
& \geq\|h\|_{H^{-1 / 4}}^{2}
\end{aligned}
$$

More information on such techniques can be found in [42] or posterior works.

\subsection{Positivity of the smooth part}

To conclude the proof of Lemma 12, we show that the smooth part of our kernel is of positive type. We could also rely on smoothness arguments to prove that its behavior doesn't modify the asymptotic behavior of eigenvectors and eigenvalues of the singular part.

Lemma 16. For any $h \in L^{2}(0,1)$,

$$
\int_{0}^{1} \int_{0}^{1}(x+y)^{-\frac{1}{2}} h(x) h(y) \mathrm{d} x \mathrm{~d} y \geq 0 .
$$

Proof. We use definitions and theorems found in [9, Chapter 3]. Thanks to [9, result 1.9, page 69], the kernel given on $(0,1)^{2}$ by $(x, y) \mapsto x+y$ is conditionnaly negative semidefinite (cnsd). Hence, using [9, corollary 2.10, page 78], the kernel given by $(x, y) \mapsto \sqrt{x+y}$ is also cnsd. Eventually, [9, exercise 2.21, page 80] proves that the kernel $(x, y) \mapsto 1 / \sqrt{x+y}$ is positive semidefinite. This means that, for any $n>0$ and any $c_{1}, \ldots c_{n} \in \mathbb{R}$ and any $x_{1}, \ldots x_{n} \in(0,1)$,

$$
\sum_{i=1}^{n} \sum_{j=1}^{n} \frac{c_{i} c_{j}}{\sqrt{x_{i}+x_{j}}} \geq 0
$$

Using Mercer's theorem (see [36]), we deduce that, for any $h \in L^{2}(0,1)$,

$$
\int_{0}^{1} \int_{0}^{1}(x+y)^{-\frac{1}{2}} h(x) h(y) \mathrm{d} x \mathrm{~d} y \geq 0 .
$$

\subsection{Conclusion of the proof}

Now we can prove Lemma 12. Indeed, combining Lemmas 14,15 and 16 proves that there exists $C>0$ such that, for any $f \in L^{2}(0,1)$,

$$
(N f, f) \geq C\|F\|_{H^{-1 / 4}(0,1)}^{2},
$$

where $F$ is the primitive of $f$ such that $F(1)=0$. Thanks to the change of variables already mentionned, the same property holds true for $K_{0}$ with the symmetrical condition $F(0)=0$. 


\section{Exact computation of the kernel and estimation of residues}

In this section, we give a detailed and rigorous expansion of the main kernel $K^{\varepsilon}$. Our goal is to be able to estimate with precision the size and the smoothness of all the residues that build up the difference between the asymptotic kernel $\sqrt{\varepsilon} K^{0}$ and the true kernel. As above, we write:

$$
\begin{aligned}
K^{\varepsilon}\left(s_{1}, s_{2}\right) & =\int_{s_{1} \vee s_{2}}^{1} A\left(t, s_{1}, s_{2}\right) \mathrm{d} t, \quad \text { where } \\
A\left(t, s_{1}, s_{2}\right) & =\int_{0}^{\frac{1}{2}} \Phi_{x}(1-t, x) G\left(t-s_{1}, x\right) G\left(t-s_{2}, x\right) \mathrm{d} x .
\end{aligned}
$$

In equations (5.1) and (5.2), it is implicit that $A, \Phi_{x}$ and $G$ depend on $\varepsilon$. Moreover, in equation (5.2), we use the fact that $G$ and $\Phi_{x}$ are even to write the integral over $x \in\left(0, \frac{1}{2}\right)$. This breaks the symmetry but will allow us to use a one-sided expansion of $G$, thereby focusing on its behavior near $x=0$.

\subsection{Smoothness of weakly singular integral operators}

We know that the asymptotic kernel $K^{0}$ is coercive with respect to the $H^{-5 / 4}$ norm of the control $u$. Thus, in order for the full kernel to remain coercive for $\varepsilon>0$, we need to prove that the residues can be bounded with the same norm. In this paragraph, we give conditions on a kernel residue $L$ that are easy to check and imply that:

$$
\forall u \in L^{2}(0,1), \quad|\langle L u, u\rangle| \lesssim\|U\|_{H^{-1 / 4}(0,1)}^{2},
$$

where $U$ is the primitive of $u$ such that $U(0)=0$. In the following paragraphs, we will check that these conditions are satisfied by our residues. We start with the following lemma, which allows us to express $\langle L u, u\rangle$ directly as a function of $U$.

Lemma 17. Let $\Gamma$ be the triangular domain $\{(x, y) \in(0,1) \times(0,1)$, s.t. $x \leq y\}$. Let $L \in W^{2,1}(\Gamma)$. We see $L$ as the restriction to $\Gamma$ of a symmetric kernel on $(0,1) \times(0,1)$ that is smooth on each triangle but not necessarly accross the first diagonal. Assume that $L(\cdot, 1) \equiv 0$. Let $u \in L^{2}(0,1)$ and $U$ be the primitive of $u$ such that $U(0)=0$. Then:

$$
\int_{\Gamma} L(x, y) u(x) u(y) \mathrm{d} x \mathrm{~d} y=\int_{\Gamma} \partial_{12} L(x, y) U(x) U(y) \mathrm{d} x \mathrm{~d} y+\frac{1}{2} \int_{0}^{1}\left(\partial_{1} L-\partial_{2} L\right)(x, x) U^{2}(x) \mathrm{d} x .
$$

In equation (5.4), it is worth to be noted that $\partial_{1} L$ and $\partial_{2} L$ are evaluated on the first diagonal and must thus be computed using points within $\Gamma$.

Proof. We use integration by parts and the boundary conditions $U(0)=0$ and $L(\cdot, 1)=0$.

$$
\begin{aligned}
\int_{\Gamma} L(x, y) u(x) u(y) \mathrm{d} x \mathrm{~d} y= & \int_{0}^{1} u(x) \int_{x}^{1} L(x, y) u(y) \mathrm{d} y \mathrm{~d} x \\
= & \int_{0}^{1} u(x)\left([L(x, y) U(y)]_{x}^{1}-\int_{x}^{1} \partial_{2} L(x, y) U(y) \mathrm{d} y\right) \mathrm{d} x \\
= & -\int_{0}^{1} L(x, x) U(x) u(x) \mathrm{d} x-\int_{0}^{1} U(y) \int_{0}^{y} \partial_{2} L(x, y) u(x) \mathrm{d} x \\
= & \int_{0}^{1} \frac{\mathrm{d}}{\mathrm{d} x}\{L(x, x)\} \cdot \frac{U^{2}}{2}(x) \mathrm{d} x \\
& -\int_{0}^{1} U(y)\left(\left[U(x) \partial_{2} L(x, y)\right]_{0}^{y}-\int_{0}^{y} \partial_{12} L(x, y) U(x) \mathrm{d} x\right) \mathrm{d} y \\
= & \int_{\Gamma} \partial_{12} L(x, y) U(x) U(y) \mathrm{d} x \mathrm{~d} y+\frac{1}{2} \int_{0}^{1}\left(\partial_{1} L-\partial_{2} L\right)(x, x) U^{2}(x) \mathrm{d} x .
\end{aligned}
$$

Equation chain (5.5) concludes the proof of equation (5.4).

Equation (5.4) includes a boundary term evaluated on the diagonal, which looks like the $L^{2}$ norm of $U$. This would forbid us to prove any estimate like (5.3). However, all our kernel residues satisfy the condition $\partial_{1} L-\partial_{2} L=0$ along the diagonal and this term thus vanishes. Hence, our task is to check that the new kernel $\partial_{12} L$ generates a bounded quadratic form on $H^{-1 / 4}(0,1)$. 
Lemma 18. Let $L$ be a continuous function defined on $\Omega=\{(x, y) \in(0,1) \times(0,1)$, s.t. $x \neq y\}$. Assume that there exists $\kappa>0$ and $\frac{1}{2}<\delta \leq 1$, such that, on $\Omega$ :

$$
\begin{gathered}
|L(x, y)| \leq \kappa|x-y|^{-\frac{1}{2}} \\
\left|L(x, y)-L\left(x^{\prime}, y\right)\right| \leq \kappa\left|x-x^{\prime}\right|^{\delta}|x-y|^{-\frac{1}{2}-\delta}, \quad \text { for }\left|x-x^{\prime}\right| \leq \frac{1}{2}|x-y|, \\
\left|L(x, y)-L\left(x, y^{\prime}\right)\right| \leq \kappa\left|y-y^{\prime}\right|^{\delta}|x-y|^{-\frac{1}{2}-\delta}, \quad \text { for }\left|y-y^{\prime}\right| \leq \frac{1}{2}|x-y|
\end{gathered}
$$

Then $L$ defines a continuous quadractic form on $H^{-1 / 4}(0,1)$. Moreover, there exists a constant $C(\delta)$ depending only on $\delta$ (and not on $L$ ) such that, for any $U \in L^{2}(0,1)$ :

$$
|\langle L U, U\rangle| \leq C(\delta) \kappa|U|_{H^{-1 / 4}(0,1)}^{2} .
$$

This technical lemma is very important for our proof because it gives a quantitative estimate, through $\kappa$, of the action of kernels against controls. This Lemma can be deduced from the works of Torres [44] and Youssfi [47]. We give a proof skeleton in Appendix A. The starting point is to prove that a kernel satisfying estimates (5.6), (5.7) and (5.8) defines a weakly singular integral operator, which is continuous from $H^{-1 / 4}$ to $H^{+1 / 4}$. Indeed, such kernels are smoother then standard CálderonZygmund operators and it is reasonable to expect that they exhibit some smoothing properties.

We end this section with two useful formulas. Let $a:(0,1)^{3} \rightarrow \mathbb{R}$ be a function such that $a\left(t, s_{1}, s_{2}\right)=a\left(t, s_{2}, s_{1}\right)$. We consider the kernel generated by $a$ :

$$
L\left(s_{1}, s_{2}\right)=\int_{s_{1} \vee s_{2}}^{1} a\left(t, s_{1}, s_{2}\right) \mathrm{d} t .
$$

Lemma 17 can be applied to such kernels because they satisfy the condition $L(\cdot, 1) \equiv 0$. We compute:

$$
\begin{aligned}
\partial_{1} L(s, s)-\partial_{2} L(s, s) & =a(s, s, s), & \text { for } s \in(0,1) \\
\partial_{12} L\left(s_{1}, s_{2}\right) & =-\partial_{s_{1}} a\left(s_{2}, s_{1}, s_{2}\right)+\int_{s_{2}}^{T} \partial_{s_{1}} \partial_{s_{2}} a\left(t, s_{1}, s_{2}\right) \mathrm{d} t, & \text { for } s_{1}<s_{2}
\end{aligned}
$$

Formulas (5.11) and (5.12) will be used extensively in the following sections. Moreover, as soon as $a(s, s, s) \equiv 0$, we see that the boundary term $\partial_{1} L-\partial_{2} L$ vanishes.

\subsection{Asymptotic expansion of $K^{\varepsilon}$}

In this section, we make our rough expansions more precise. Therefore we decompose $G$ and $\Phi$ using the same first order terms as for the heuristic, but this time we introduce and compute the residues.

\subsubsection{Expansion of $G$ as $\varepsilon \rightarrow 0$}

Recall that we only need to approximate $G$ for $x \in(0,1 / 2)$. Keeping our approximation introduced in (3.16), we expand $G$ as:

$$
G(t, x)=\operatorname{erf}\left(\frac{x}{\sqrt{4 \varepsilon t}}\right)+H(t, x),
$$

where $H \in \mathcal{C}^{\infty}((0,1) \times(0,1 / 2))$ is the solution to:

$$
\left\{\begin{aligned}
H_{t}-\varepsilon H_{x x} & =0 & & \text { in }(0,1) \times(0,1 / 2), \\
H(t, 0) & =0 & & \text { in }(0,1), \\
H_{x}(t, 1 / 2) & =\sigma(\varepsilon t) & & \text { in }(0,1), \\
H(0, x) & =0 & & \text { in }(0,1 / 2),
\end{aligned}\right.
$$

where the source term $\sigma$ comes from the boundary condition $G_{x}(t, 1 / 2)=0$ and balances out the trace of the erf() part:

$$
\sigma(s)=-\left.\frac{\partial}{\partial x}\left[\operatorname{erf}\left(\frac{x}{\sqrt{4 s}}\right)\right]\right|_{x=\frac{1}{2}}=-\frac{1}{\sqrt{s \pi}} \exp \left(-\frac{1}{16 s}\right) .
$$


Lemma 19. Let $0<\gamma<\frac{1}{16}$. There exists $C(\gamma)>0$ such that:

$$
\left\|H_{t}\right\|_{\infty}+\left\|H_{t x}\right\|_{\infty}+\left\|H_{t t}\right\|_{\infty}+\left\|H_{t t x}\right\|_{\infty} \leq C(\gamma) e^{-\gamma / \varepsilon} .
$$

Proof. This lemma is due to the exponentially decaying factor within the source term $\sigma$ defined by (5.15), which allows as many differentiations with respect to $x$ or $t$ as needed to be done. Estimate (5.16) could in fact be derived for further derivatives. Let us give a sketch of proof.

First, note that $H^{(3)}:=H_{t t t}$ is the solution to a similar system as (5.14) with the boundary condition $H_{x}^{(3)}(t, 1 / 2)=\varepsilon^{3} \sigma^{(3)}(\varepsilon t)$. We can convert this boundary condition into a source term by writing $H^{(3)}(t, x)=x \varepsilon^{3} \sigma^{(3)}(\varepsilon t)+\tilde{H}^{(3)}$, where $\tilde{H}^{(3)}$ is now the solution to a heat equation with homogeneous mixed boundary conditions and a source term $-x \varepsilon^{4} \sigma^{(4)}(\varepsilon t)$. Applying the maximum principle yields an estimate of the form $\left\|\tilde{H}^{(3)}\right\|_{\infty} \leq C(\gamma) e^{-\gamma / \varepsilon}$. Since $\varepsilon H_{t t x x}=H^{(3)}$, we obtain an $L^{\infty}$ estimate of the same form for $H_{t t x x}$. By integration with respect to time and space, we obtain (5.16).

\subsubsection{Expansion of $\Phi$ as $\varepsilon \rightarrow 0$}

Guided by our rough computations, we decompose $\Phi \in X_{1}$, the solution to (3.4) as:

$$
\Phi(t, x)=\rho(x)+\varepsilon \phi(t, x) .
$$

Thus, we introduce the partial differential equation satisfied by $\phi \in X_{1}$ :

$$
\left\{\begin{aligned}
\phi_{t}-\varepsilon \phi_{x x}=\rho_{x x} & & \text { in }(0,1) \times(0,1), \\
\phi(t, 0)=0 & & \text { in }(0,1), \\
\phi(t, 1)=0 & & \text { in }(0,1), \\
\phi(0, x)=0 & & \text { in }(0,1) .
\end{aligned}\right.
$$

Lemma 20. The following estimates hold:

$$
\begin{aligned}
\left\|\Phi_{x}\right\|_{\infty} & \lesssim 1, \\
\left\|\phi_{x}\right\|_{\infty} & \lesssim 1, \\
\left\|\Phi_{t x}\right\|_{\infty}=\left\|\varepsilon \phi_{t x}\right\|_{\infty} & \lesssim \varepsilon .
\end{aligned}
$$

Proof. Estimates (5.19), (5.20) and (5.21) can be proved using a Fourier series decomposition for heat equations. As an example, let us prove (5.21). We introduce the basis $e_{n}(x)=\sqrt{2} \sin (n \pi x)$. Since $\phi_{t}$ is the solution to a heat equation with initial data $\rho_{x x} \in H_{0}^{1}$, we have:

$$
\phi_{t}(t, x)=\sum_{n=1}^{+\infty} e^{-\varepsilon n^{2} \pi^{2} t}\left\langle\rho_{x x}, e_{n}\right\rangle e_{n}(x) .
$$

Thanks to the choice of $\rho$ in $(3.14)$, we have $\rho_{x x}(0)=\rho_{x x}(1)=0$. Thus,

$$
\left\langle\rho_{x x}, e_{n}\right\rangle=-\frac{1}{n^{2} \pi^{2}}\left\langle\rho_{x x x x}, e_{n}\right\rangle=\frac{12 \sqrt{2}}{n^{3} \pi^{3}}\left((-1)^{n}-1\right)=\mathcal{O}\left(\frac{1}{n^{3}}\right) .
$$

Combining equations (5.22) and (5.23) yields:

$$
\left\|\phi_{t x}\right\|_{\infty} \leq \sum_{n=1}^{+\infty} n \pi\left|\left\langle\rho_{x x}, e_{n}\right\rangle\right| \lesssim \sum_{n=1}^{+\infty} \frac{1}{n^{2}} .
$$

Equation (5.24) concludes the proof of (5.21). A similar method can be applied to prove (5.19) and (5.20). 


\subsubsection{Five stages expansion of the full kernel}

Using expansions (5.13) and (5.17), and the fact that $\int \Phi_{x}=0$, we break down the generator $A\left(t, s_{1}, s_{2}\right)$ into 6 smaller kernel generators, $A_{1}$ through $A_{6}$, defined by:

$$
\begin{aligned}
& A_{1}\left(t, s_{1}, s_{2}\right)=\int_{0}^{\frac{1}{2}} \rho_{x}(0)\left(\operatorname{erf}\left(\frac{x}{\sqrt{4 \varepsilon\left(t-s_{1}\right)}}\right) \operatorname{erf}\left(\frac{x}{\sqrt{4 \varepsilon\left(t-s_{2}\right)}}\right)-1\right) \mathrm{d} x \\
& A_{2}\left(t, s_{1}, s_{2}\right)=\int_{0}^{\frac{1}{2}}\left(\rho_{x}(x)-\rho_{x}(0)\right)\left(\operatorname{erf}\left(\frac{x}{\sqrt{4 \varepsilon\left(t-s_{1}\right)}}\right) \operatorname{erf}\left(\frac{x}{\sqrt{4 \varepsilon\left(t-s_{2}\right)}}\right)-1\right) \mathrm{d} x \\
& A_{3}\left(t, s_{1}, s_{2}\right)=\int_{0}^{\frac{1}{2}} \varepsilon \phi_{x}(1-t, x)\left(\operatorname{erf}\left(\frac{x}{\sqrt{4 \varepsilon\left(t-s_{1}\right)}}\right) \operatorname{erf}\left(\frac{x}{\sqrt{4 \varepsilon\left(t-s_{2}\right)}}\right)-1\right) \mathrm{d} x \\
& A_{4}\left(t, s_{1}, s_{2}\right)=\int_{0}^{\frac{1}{2}} \Phi_{x}(1-t, x) H\left(t-s_{1}, x\right) \operatorname{erf}\left(\frac{x}{\sqrt{4 \varepsilon\left(t-s_{2}\right)}}\right) \mathrm{d} x \\
& A_{5}\left(t, s_{1}, s_{2}\right)=\int_{0}^{\frac{1}{2}} \Phi_{x}(1-t, x) H\left(t-s_{2}, x\right) \cdot \operatorname{erf}\left(\frac{x}{\sqrt{4 \varepsilon\left(t-s_{1}\right)}}\right) \mathrm{d} x \\
& A_{6}\left(t, s_{1}, s_{2}\right)=\int_{0}^{\frac{1}{2}} \Phi_{x}(1-t, x) H\left(t-s_{1}, x\right) H\left(t-s_{2}, x\right) \mathrm{d} x
\end{aligned}
$$

It can be checked that $A$ defined in (5.2) is indeed equal to the sum of $A_{1}$ through $A_{6}$. For each $1 \leq i \leq 6$, we consider the associated kernel generated by $A_{i}$ :

$$
K_{i}\left(t, s_{1}, s_{2}\right)=\int_{s_{1} \vee s_{2}}^{T} A_{i}\left(t, s_{1}, s_{2}\right) \mathrm{d} t .
$$

A first remark is that, for each $1 \leq i \leq 6, A_{i}(s, s, s) \equiv 0$ on $(0,1)$. Thus, equation (5.11) tells us that there will be no boundary term involving $|u|_{H^{-1}}$.

\subsubsection{Proof methodology}

The six following paragraphs are dedicated to estimates for $K_{1}$ through $K_{6}$. In order to organize the computations that will be carried out for each of these six kernels, we introduce the notations:

$$
\begin{aligned}
T_{i}\left(s_{1}, s_{2}\right) & =\left.\frac{\partial A_{i}}{\partial s_{1}}\left(t, s_{1}, s_{2}\right)\right|_{t=s_{2}}, \\
Q_{i}\left(t, s_{1}, s_{2}\right) & =\frac{\partial^{2} A_{i}}{\partial s_{1} \partial s_{2}}\left(t, s_{1}, s_{2}\right), \\
R_{i}\left(s_{1}, s_{2}\right) & =\int_{s_{2}}^{1} Q_{i}\left(t, s_{1}, s_{2}\right) \mathrm{d} t .
\end{aligned}
$$

Using formula (5.12), $\partial_{12} K_{i}=R_{i}-T_{i}$. Therefore, thanks to Lemma 18 and Lemma 17, we need to prove that each $T_{i}$ and each $R_{i}$ satisfies the conditions (5.6), (5.7) and (5.8). For a kernel $L$, we will denote $\kappa(L)$ the associated constant in Lemma 18. In the following paragraphs, we investigate the behavior of $\kappa\left(\partial_{12} K_{i}\right)$ with respect to $\varepsilon$. We end this paragraph with a useful estimation lemma.

Lemma 21. For any $k>0$ there exists $c_{k}>0$ such that, for any $\lambda>0$, for any $\varepsilon>0$,

$$
\int_{0}^{+\infty} x^{k} \exp \left(-\frac{x^{2}}{4 \varepsilon \lambda}\right) \mathrm{d} x \leq c_{k}(\varepsilon \lambda)^{\frac{k+1}{2}} .
$$

Proof. Use a change of variables introducing $\tilde{x}=x / \sqrt{4 \varepsilon \lambda}$.

In the following paragraphs, similarly as we use the $\lesssim$ sign, we will use the $\approx$ sign to denote equalities that hold up to a numerical constant (independent on all variables) of which we will not keep track. 


\subsection{Handling the $K_{1}$ kernel}

The kernel $K_{1}$ contains the main coercive part of $K^{\varepsilon}$ discovered in Section 3. Starting from its definition in (5.25), we decompose it using a scaling on $x$ :

$$
\begin{aligned}
A_{1}\left(t, s_{1}, s_{2}\right) & =\rho_{x}(0) \int_{0}^{\frac{1}{2}}\left(\operatorname{erf}\left(\frac{x}{\sqrt{4 \varepsilon\left(t-s_{1}\right)}}\right) \operatorname{erf}\left(\frac{x}{\sqrt{4 \varepsilon\left(t-s_{2}\right)}}\right)-1\right) \mathrm{d} x \\
& =\frac{\sqrt{\varepsilon}}{15} \int_{0}^{\frac{1}{4 \sqrt{\varepsilon}}}\left(1-\operatorname{erf} \frac{x}{\sqrt{\alpha}} \operatorname{erf} \frac{x}{\sqrt{\beta}}\right) \mathrm{d} x \\
& =\frac{\sqrt{\varepsilon}}{15} \int_{0}^{+\infty}\left(1-\operatorname{erf} \frac{x}{\sqrt{\alpha}} \operatorname{erf} \frac{x}{\sqrt{\beta}}\right) \mathrm{d} x-\frac{\sqrt{\varepsilon}}{15} \int_{\frac{1}{4 \sqrt{\varepsilon}}}^{+\infty}\left(1-\operatorname{erf} \frac{x}{\sqrt{\alpha}} \operatorname{erf} \frac{x}{\sqrt{\beta}}\right) \mathrm{d} x .
\end{aligned}
$$

The first integral gives rise to the main coercive part of the kernel and has already been computed exactly in Section 3. The second part is a residue and has to be taken care of. Let us name it $\tilde{A}_{1}$ :

$$
\tilde{A}_{1}\left(t, s_{1}, s_{2}\right)=-\frac{\sqrt{\varepsilon}}{15} \int_{\frac{1}{4 \sqrt{\varepsilon}}}^{+\infty}\left(\operatorname{erf}\left(\frac{x}{\sqrt{\alpha}}\right) \operatorname{erf}\left(\frac{x}{\sqrt{\beta}}\right)-1\right) \mathrm{d} x .
$$

Therefore, equation (5.36) yields:

$$
K_{1}\left(s_{1}, s_{2}\right)=\frac{\sqrt{\varepsilon}}{45 \sqrt{\pi}} K^{0}\left(s_{1}, s_{2}\right)+\tilde{K}_{1}\left(s_{1}, s_{2}\right) .
$$

Lemma 22. There exist $c>0$ and $\gamma>0$ such that, for any $\varepsilon>0$,

$$
\kappa\left(\partial_{12} \tilde{K}_{1}\right) \leq c \cdot \exp \left(-\frac{\gamma}{\varepsilon}\right)
$$

where $\kappa\left(\partial_{12} \tilde{K}_{1}\right)$ is the constant associated to the weakly singular integral operator $\tilde{K}_{1}$ in Lemma 18.

Proof. Recalling notations (5.32), (5.33) and (5.34), we compute:

$$
\begin{gathered}
\tilde{T}_{1}\left(s_{1}, s_{2}\right)=\left.\left(\partial_{s_{1}} \tilde{A}_{1}\right)\right|_{t=s_{2}} \approx \varepsilon^{1 / 2} \Delta^{-3 / 2} \int_{\frac{1}{4 \sqrt{\varepsilon}}}^{+\infty} x \exp \left(-\frac{x^{2}}{\Delta}\right) \mathrm{d} x \\
\tilde{Q}_{1}\left(t, s_{1}, s_{2}\right)=\partial_{s_{1}} \partial_{s_{2}} \tilde{A}_{1}\left(t, s_{1}, s_{2}\right) \approx \varepsilon^{1 / 2}(\alpha \beta)^{-3 / 2} \int_{\frac{1}{4 \sqrt{\varepsilon}}}^{+\infty} x^{2} \exp \left(-x^{2}\left(\frac{1}{\alpha}+\frac{1}{\beta}\right)\right) \mathrm{d} x \\
\tilde{R}_{1}\left(s_{1}, s_{2}\right)=\int_{s_{2}}^{1} \tilde{Q}_{1}\left(t, s_{1}, s_{2}\right) \approx \varepsilon^{1 / 2} \int_{s_{2}}^{1}(\alpha \beta)^{-3 / 2} \int_{\frac{1}{4 \sqrt{\varepsilon}}}^{+\infty} x^{2} \exp \left(-x^{2}\left(\frac{1}{\alpha}+\frac{1}{\beta}\right)\right) \mathrm{d} x \mathrm{~d} t
\end{gathered}
$$

where we introduce $\Delta=s_{2}-s_{1}$, that will also be used in the sequel. We claim that both $\tilde{T}_{1}$ and $\tilde{R}_{1}$ are $\mathcal{C}^{\infty}$ kernels on $(0,1) \times(0,1)$. Moreover, all their derivatives are bounded by $e^{-\gamma / \varepsilon}$ for any $\gamma<1 / 16$, thanks to the exponential terms in (5.40) and (5.42). We omit the detailed computations in order to focus on the tougher kernels.

\subsection{Handling the $K_{2}$ kernel}

Using the definition of $\rho$ given in (3.14), we rewrite $A_{2}$ defined in (5.26) as:

$$
\begin{aligned}
A_{2}\left(t, s_{1}, s_{2}\right) & =\int_{0}^{\frac{1}{2}}\left(\rho_{x}(x)-\rho_{x}(0)\right) \operatorname{erf}\left(\frac{x}{\sqrt{4 \varepsilon \alpha}}\right) \operatorname{erf}\left(\frac{x}{\sqrt{4 \varepsilon \beta}}\right) \mathrm{d} x \\
& =\int_{0}^{\frac{1}{2}} x^{2}(x-1)^{2} \operatorname{erf}\left(\frac{x}{\sqrt{4 \varepsilon \alpha}}\right) \operatorname{erf}\left(\frac{x}{\sqrt{4 \varepsilon \beta}}\right) \mathrm{d} x .
\end{aligned}
$$

First part. Remembering that $\operatorname{erf}(+\infty)=1$, we consider the first order derivative:

$$
T_{2}\left(s_{1}, s_{2}\right)=\left.\left(\partial_{s_{1}} A_{2}\right)\right|_{t=s_{2}} \approx \varepsilon^{-1 / 2} \Delta^{-3 / 2} \int_{0}^{\frac{1}{2}} x^{3}(x-1)^{2} \exp \left(-\frac{x^{2}}{4 \varepsilon \Delta}\right) \mathrm{d} x
$$


Using Lemma 21 and differentiating gives:

$$
\begin{aligned}
\left|T_{2}\left(s_{1}, s_{2}\right)\right| & \lesssim \varepsilon^{3 / 2} \Delta^{1 / 2}, \\
\left|\partial_{s_{1}} T_{2}\left(s_{1}, s_{2}\right)\right| & \lesssim \varepsilon^{3 / 2} \Delta^{-1 / 2}, \\
\left|\partial_{s_{2}} T_{2}\left(s_{1}, s_{2}\right)\right| & \lesssim \varepsilon^{3 / 2} \Delta^{-1 / 2} .
\end{aligned}
$$

Estimates (5.45) prove that $\kappa\left(T_{2}\right) \lesssim \varepsilon^{3 / 2}$. In fact, $T_{2}$ is a smoother than the weakly singular integral operators studied in Lemma 18, since such operators allow degeneracy like $\Delta^{-1 / 2}$ along the diagonal. Moreover, we proved that $T_{2}$ is Lipschitz continuous, whereas Lemma 18 only requires $\mathcal{C}^{p}$ with $p>\frac{1}{2}$.

Second part. Now we consider the second order derivative. Let us compute:

$$
Q_{2}\left(t, s_{1}, s_{2}\right)=\partial_{s_{1}} \partial_{s_{2}} A_{2}\left(t, s_{1}, s_{2}\right) \approx \varepsilon^{-1}(\alpha \beta)^{-3 / 2} \int_{0}^{\frac{1}{2}} x^{4}(x-1)^{2} \exp \left(-\frac{x^{2}}{4 \varepsilon}\left(\frac{1}{\alpha}+\frac{1}{\beta}\right)\right) \mathrm{d} x .
$$

Thanks to Lemma 21, we estimate the size of $Q_{2}$ :

$$
\left|Q_{2}\left(t, s_{1}, s_{2}\right)\right| \lesssim \varepsilon^{3 / 2}(\alpha \beta)^{-3 / 2}\left(\frac{1}{\alpha}+\frac{1}{\beta}\right)^{-5 / 2}=\frac{\varepsilon^{3 / 2} \alpha \beta}{(\alpha+\beta)^{5 / 2}}
$$

Writing $\alpha=\Delta+\tau$ and $\beta=\tau$, we can estimate:

$$
\left|R_{2}\left(s_{1}, s_{2}\right)\right|=\left|\int_{s_{2}}^{1} Q_{2}\left(t, s_{1}, s_{2}\right) \mathrm{d} t\right| \lesssim \varepsilon^{3 / 2} \int_{0}^{1} \frac{\tau(\Delta+\tau)}{(\Delta+2 \tau)^{5 / 2}} \mathrm{~d} \tau \lesssim \varepsilon^{3 / 2} \Delta^{-1 / 2} .
$$

We should now move on to computing $\partial_{s_{1}} R_{2}$ and $\partial_{s_{2}} R_{2}$, to establish the missing estimates on $R_{2}$. However, the computations associated to $R_{2}$ are very similar to the ones that we carry out for $R_{3}$. Since $R_{3}$ is a little harder, we skip the proof for $R_{2}$ and refer the reader to the proof of $R_{3}$, which is fully detailed in the next paragraph. Therefore, we claim that:

$$
\kappa\left(\partial_{12} K_{2}\right) \lesssim \varepsilon^{3 / 2} \text {. }
$$

\subsection{Handling the $K_{3}$ kernel}

In this section, we consider:

$$
A_{3}\left(t, s_{1}, s_{2}\right)=\varepsilon \int_{0}^{\frac{1}{2}} \phi_{x}(1-t, x)\left(\operatorname{erf}\left(\frac{x}{\sqrt{4 \varepsilon\left(t-s_{1}\right)}}\right) \operatorname{erf}\left(\frac{x}{\sqrt{4 \varepsilon\left(t-s_{2}\right)}}\right)-1\right) \mathrm{d} x .
$$

First part. Remembering that $\operatorname{erf}(+\infty)=1$, we consider the first order derivative:

$$
T_{3}\left(s_{1}, s_{2}\right):=\left.\left(\partial_{s_{1}} A_{3}\right)\right|_{t=s_{2}} \approx \varepsilon^{1 / 2} \Delta^{-3 / 2} \int_{0}^{\frac{1}{2}} \phi_{x}\left(1-s_{2}, x\right) \cdot x \exp \left(-\frac{x^{2}}{4 \varepsilon \Delta}\right) \mathrm{d} x .
$$

Thanks to Lemma 20 and Lemma 21, we have

$$
\left|T_{3}\left(s_{1}, s_{2}\right)\right| \lesssim \varepsilon^{1 / 2} \Delta^{-3 / 2}\left\|\phi_{x}\right\|_{\infty} \cdot \int_{0}^{\frac{1}{2}} x \exp \left(-\frac{x^{2}}{4 \varepsilon \Delta}\right) \mathrm{d} x \lesssim \varepsilon^{3 / 2} \Delta^{-1 / 2}
$$

Moreover,

$$
\begin{aligned}
\left|\partial_{s_{1}} T_{3}\left(s_{1}, s_{2}\right)\right| \lesssim & \varepsilon^{1 / 2} \Delta^{-5 / 2}\left\|\phi_{x}\right\|_{\infty} \cdot \int_{0}^{\frac{1}{2}} x \exp \left(-\frac{x^{2}}{4 \varepsilon \Delta}\right) \mathrm{d} x \\
& +\varepsilon^{1 / 2} \Delta^{-3 / 2}\left\|\phi_{x}\right\|_{\infty} \cdot \int_{0}^{\frac{1}{2}} \frac{x^{3}}{4 \varepsilon \Delta^{2}} \exp \left(-\frac{x^{2}}{4 \varepsilon \Delta}\right) \mathrm{d} x \\
\lesssim & \varepsilon^{3 / 2} \Delta^{-3 / 2}
\end{aligned}
$$

and

$$
\begin{aligned}
\left|\partial_{s_{2}} T_{3}\left(s_{1}, s_{2}\right)\right| \lesssim & \varepsilon^{1 / 2} \Delta^{-3 / 2}\left\|\phi_{x t}\right\|_{\infty} \cdot \int_{0}^{\frac{1}{2}} x \exp \left(-\frac{x^{2}}{4 \varepsilon \Delta}\right) \mathrm{d} x \\
& +\varepsilon^{1 / 2} \Delta^{-5 / 2}\left\|\phi_{x}\right\|_{\infty} \cdot \int_{0}^{\frac{1}{2}} x \exp \left(-\frac{x^{2}}{4 \varepsilon \Delta}\right) \mathrm{d} x \\
& +\varepsilon^{1 / 2} \Delta^{-3 / 2}\left\|\phi_{x}\right\|_{\infty} \cdot \int_{0}^{\frac{1}{2}} \frac{x^{3}}{4 \varepsilon \Delta^{2}} \exp \left(-\frac{x^{2}}{4 \varepsilon \Delta}\right) \mathrm{d} x \\
\lesssim & \varepsilon^{3 / 2} \Delta^{-3 / 2} .
\end{aligned}
$$


Putting together estimates (5.51), (5.52) and (5.53) proves that $\kappa\left(T_{3}\right) \lesssim \varepsilon^{3 / 2}$.

Second part. Let us move on to the second order derivative part. We compute:

$$
Q_{3}\left(t, s_{1}, s_{2}\right)=\partial_{s_{1}} \partial_{s_{2}} A_{3} \approx(\alpha \beta)^{-3 / 2} \int_{0}^{\frac{1}{2}} x^{2} \phi_{x}(1-t, x) \exp \left(-\frac{x^{2}}{4 \varepsilon}\left(\frac{1}{\alpha}+\frac{1}{\beta}\right)\right) \mathrm{d} x .
$$

Combining Lemma 21 and Lemma 20 yields:

$$
\left|Q_{3}\left(t, s_{1}, s_{2}\right)\right| \lesssim \frac{\varepsilon^{3 / 2}}{(\alpha+\beta)^{3 / 2}} .
$$

Writing $\alpha=\Delta+\tau$ and $\beta=\tau$, we can estimate:

$$
\left|R_{3}\left(s_{1}, s_{2}\right)\right|=\left|\int_{s_{2}}^{1} Q_{3}\left(t, s_{1}, s_{2}\right) \mathrm{d} t\right| \lesssim \int_{0}^{1}\left(\frac{\varepsilon}{\Delta+2 \tau}\right)^{3 / 2} \mathrm{~d} \tau \lesssim \varepsilon^{3 / 2} \Delta^{-1 / 2} .
$$

Now we will prove similar estimates for the first order derivatives of $R_{3}$. Differentiating equation (5.54) with respect to $s_{1}$ (or similarly $\alpha$ ) yields:

$$
\begin{aligned}
\partial_{s_{1}} Q_{3}\left(t, s_{1}, s_{2}\right) \approx & -\frac{3}{2} \alpha^{-5 / 2} \beta^{-3 / 2} \int_{0}^{\frac{1}{2}} x^{2} \phi_{x}(1-t, x) \exp \left(-\frac{x^{2}}{4 \varepsilon}\left(\frac{1}{\alpha}+\frac{1}{\beta}\right)\right) \mathrm{d} x \\
& +(\alpha \beta)^{-3 / 2} \frac{1}{\alpha^{2}} \int_{0}^{\frac{1}{2}} \frac{x^{4}}{4 \varepsilon} \phi_{x}(1-t, x) \exp \left(-\frac{x^{2}}{4 \varepsilon}\left(\frac{1}{\alpha}+\frac{1}{\beta}\right)\right) \mathrm{d} x .
\end{aligned}
$$

Combining Lemma 21 and Lemma 20 gives:

$$
\left|\partial_{s_{1}} Q_{3}\left(t, s_{1}, s_{2}\right)\right| \lesssim \alpha^{-5 / 2} \beta^{-3 / 2} \frac{\varepsilon^{3 / 2}}{\left(\frac{1}{\alpha}+\frac{1}{\beta}\right)^{3 / 2}}+\alpha^{-7 / 2} \beta^{-3 / 2} \frac{\varepsilon^{3 / 2}}{\left(\frac{1}{\alpha}+\frac{1}{\beta}\right)^{5 / 2}} \lesssim \varepsilon^{3 / 2} \alpha^{-5 / 2} .
$$

Integration with respect to $t$ yields an estimate of $\partial_{s_{1}} R_{3}$ :

$$
\left|\partial_{s_{1}} R_{3}\left(s_{1}, s_{2}\right)\right| \lesssim \int_{s_{2}}^{1}\left|\partial_{s_{1}} Q_{3}\left(t, s_{1}, s_{2}\right)\right| \mathrm{d} t \lesssim \varepsilon^{3 / 2} \int_{s_{2}}^{1} \frac{\mathrm{d} t}{\alpha^{5 / 2}} \lesssim \varepsilon^{3 / 2} \Delta^{-3 / 2} .
$$

From this, we deduce that:

$$
\left|R_{3}\left(s_{1}, s_{2}\right)-R_{3}\left(\tilde{s_{1}}, s_{2}\right)\right| \lesssim \varepsilon^{3 / 2} \Delta^{-3 / 2}\left|s_{1}-\tilde{s_{1}}\right| .
$$

Eventually, we finish with the smoothness of $R_{3}$ with respect to $s_{2}$. We compute the difference for $s_{1}<s_{2}<\tilde{s_{2}}$ with $\tilde{s_{2}}-s_{2} \leq \frac{1}{2}\left(s_{2}-s_{1}\right)$ :

$$
\begin{aligned}
\left|R_{3}\left(s_{1}, s_{2}\right)-R_{3}\left(s_{1}, \tilde{s_{2}}\right)\right| & =\left|\int_{s_{2}}^{1} Q_{3}\left(t, s_{1}, s_{2}\right) \mathrm{d} t-\int_{\tilde{s_{2}}}^{1} Q_{3}\left(t, s_{1}, \tilde{s_{2}}\right) \mathrm{d} t\right| \\
& =\left|\int_{s_{2}}^{\tilde{s_{2}}} Q_{3}\left(t, s_{1}, s_{2}\right) \mathrm{d} t-\int_{\tilde{s_{2}}}^{1}\left(Q_{3}\left(t, s_{1}, \tilde{s_{2}}\right)-Q_{3}\left(t, s_{1}, s_{2}\right)\right) \mathrm{d} t\right| \\
& \leq \int_{s_{2}}^{\tilde{s_{2}}} \frac{\varepsilon^{3 / 2}}{\Delta^{3 / 2}} \mathrm{~d} t+\left|\int_{\tilde{s_{2}}}^{1} \int_{s_{2}}^{\tilde{s_{2}}} \partial_{s_{2}} Q_{3}\left(t, s_{1}, s\right) \mathrm{d} s \mathrm{~d} t\right| \\
& \leq \frac{\varepsilon^{3 / 2}}{\Delta^{3 / 2}}\left|s_{2}-\tilde{s_{2}}\right|+\int_{s_{2}}^{\tilde{s}} \int_{\tilde{s_{2}}}^{1}\left|\partial_{s_{2}} Q_{3}\left(t, s_{1}, s\right)\right| \mathrm{d} t \mathrm{~d} s .
\end{aligned}
$$

The first term is already in the correct form. We need to work on the second term. Proceeding as above, differentiating equation (5.54) with respect to $s_{2}$ (or similarly $\beta$ ), then combining Lemma 21 and Lemma 20 gives:

$$
\left|\partial_{s_{2}} Q_{3}\left(t, s_{1}, s\right)\right| \lesssim \varepsilon^{3 / 2} \frac{1}{t-s} \frac{1}{\left(t-s+t-s_{1}\right)^{3 / 2}} .
$$


We compute:

$$
\begin{aligned}
\int_{\tilde{s_{2}}}^{\tilde{s_{2}}} \int_{\tilde{s_{2}}}^{1}\left|\partial_{s_{2}} Q_{3}\left(t, s_{1}, s\right)\right| \mathrm{d} t \mathrm{~d} s & \leq \varepsilon^{3 / 2} \int_{s_{2}}^{\tilde{s_{2}}} \int_{\tilde{s_{2}}}^{1} \frac{1}{t-s} \frac{1}{\left(t-s_{1}\right)^{3 / 2}} \mathrm{~d} t \mathrm{~d} s \\
& \leq \varepsilon^{3 / 2} \Delta^{-3 / 2} \int_{s_{2}}^{\tilde{s_{2}}} \int_{\tilde{s_{2}}}^{1} \frac{\mathrm{d} t}{t-s} \mathrm{~d} s \\
& \leq \varepsilon^{3 / 2} \Delta^{-3 / 2} \int_{s_{2}}^{\tilde{s_{2}}}\left|\ln \left(\tilde{s_{2}}-s\right)\right| \mathrm{d} s \\
& \leq \varepsilon^{3 / 2} \Delta^{-3 / 2}\left|s_{2}-\tilde{s_{2}}\right|\left(1+\ln \left|s_{2}-\tilde{s_{2}}\right|\right) .
\end{aligned}
$$

This last estimate does not give Lipschitz smoothness, but it does provide $\mathcal{C}^{p}$ smoothness for any $p<1$, which is enough. Together, estimates (5.56), (5.60) and (5.63) prove that $\kappa\left(R_{3}\right) \lesssim \varepsilon^{3 / 2}$.

\subsection{Handling the $K_{4}$ kernel}

In this section, we consider:

$$
A_{4}\left(t, s_{1}, s_{2}\right)=\int_{0}^{\frac{1}{2}} \Phi_{x}(1-t, x) H\left(t-s_{1}, x\right) \operatorname{erf}\left(\frac{x}{\sqrt{4 \varepsilon\left(t-s_{2}\right)}}\right) \mathrm{d} x .
$$

First part. We consider the first order derivative:

$$
\begin{aligned}
T_{4}\left(s_{1}, s_{2}\right) & =\left.\left(\partial_{s_{1}} A_{4}\right)\right|_{t=s_{2}} \\
& =\int_{0}^{\frac{1}{2}} \Phi_{x}\left(1-s_{2}, x\right) H_{t}\left(s_{2}-s_{1}, x\right) \mathrm{d} x,
\end{aligned}
$$

where we used the fact that $\operatorname{erf}(+\infty)=1$. The following estimates are straight forward:

$$
\begin{aligned}
\left|T_{2}\left(s_{1}, s_{2}\right)\right| \leq & \left\|\Phi_{x}\right\|_{\infty}\left\|H_{t}\right\|_{\infty} \\
\left|T_{2}\left(s_{1}, s_{2}\right)-T_{2}\left(\tilde{s}_{1}, s_{2}\right)\right| \leq & \left|s_{1}-\tilde{s}_{1}\right| \cdot\left\|\Phi_{x}\right\|_{\infty}\left\|H_{t t}\right\|_{\infty} \\
\left|T_{2}\left(s_{1}, s_{2}\right)-T_{2}\left(s_{1}, \tilde{s}_{2}\right)\right| \leq & \left|s_{2}-\tilde{s}_{2}\right| \cdot\left\|\Phi_{x}\right\|_{\infty}\left\|H_{t t}\right\|_{\infty} \\
& +\left|s_{2}-\tilde{s}_{2}\right| \cdot\left\|\Phi_{t x}\right\|_{\infty}\left\|H_{t}\right\|_{\infty} .
\end{aligned}
$$

Second part. We move on to the second order derivative part. We compute:

$$
Q_{4}\left(t, s_{1}, s_{2}\right)=\partial_{s_{1}} \partial_{s_{2}} A_{4}\left(t, s_{1}, s_{2}\right) \approx \varepsilon^{-1 / 2} \beta^{-3 / 2} \int_{0}^{\frac{1}{2}} x \Phi_{x}(1-t, x) H_{t}(\alpha, x) \exp \left(-\frac{x^{2}}{4 \varepsilon \beta}\right) \mathrm{d} x .
$$

Since $H_{t}(t, 0) \equiv 0,\left|H_{t}(t, x)\right| \leq x\left\|H_{t x}\right\|_{\infty}$. Using Lemma 21, we obtain:

$$
\begin{aligned}
\left|Q_{4}\left(t, s_{1}, s_{2}\right)\right| & \lesssim \varepsilon^{-1 / 2} \beta^{-3 / 2}\left\|H_{t x}\right\|_{\infty}\left\|\Phi_{x}\right\|_{\infty} \int_{0}^{\frac{1}{2}} x^{2} \exp \left(-\frac{x^{2}}{4 \varepsilon \beta}\right) \mathrm{d} x \\
& \lesssim \varepsilon\left\|H_{t x}\right\|_{\infty}\left\|\Phi_{x}\right\|_{\infty} .
\end{aligned}
$$

By integration over $t \in\left(s_{2}, 1\right)$, we obtain:

$$
\left|R_{4}\left(s_{1}, s_{2}\right)\right| \lesssim \varepsilon\left\|H_{t x}\right\|_{\infty}\left\|\Phi_{x}\right\|_{\infty} .
$$

Now we establish the smoothness of $Q_{4}$ with respect to $s_{1}$. Differentiating equation (5.69) with respect to $s_{1}$ (or $\alpha$ ), and applying the same techniques yields the estimate:

$$
\left|\partial_{s_{1}} Q_{4}\left(t, s_{1}, s_{2}\right)\right| \lesssim \varepsilon\left\|H_{t t x}\right\|_{\infty}\left\|\Phi_{x}\right\|_{\infty} .
$$

This proves that:

$$
\left|R_{4}\left(s_{1}, s_{2}\right)-R_{4}\left(\tilde{s_{1}}, s_{2}\right)\right| \lesssim \varepsilon\left\|H_{t t x}\right\|_{\infty}\left\|\Phi_{x}\right\|_{\infty} \cdot\left|s_{1}-\tilde{s_{1}}\right| \cdot
$$

Finally, we consider the smoothness of $Q_{4}$ with respect to $s_{2}$. We know that:

$$
\left|R_{4}\left(s_{1}, s_{2}\right)-R_{4}\left(s_{1}, \tilde{s_{2}}\right)\right| \leq \int_{s_{2}}^{\tilde{s_{2}}}\left|Q_{4}\left(t, s_{1}, s_{2}\right)\right| \mathrm{d} t+\int_{s_{2}}^{\tilde{s_{2}}} \int_{\tilde{s_{2}}}^{1}\left|\partial_{s_{2}} Q_{4}\left(t, s_{1}, s\right)\right| \mathrm{d} t \mathrm{~d} s .
$$


This first part obviously gives rise to a Lipschitz estimate. As for the second part, we compute $\partial_{s_{2}} Q_{4}$ by differentiating (5.69) with respect to $\beta$. We obtain

$$
\begin{aligned}
\partial_{s_{2}} Q_{4}\left(t, s_{1}, s\right)\left(t, s_{1}, s\right) \approx & -\frac{3}{2} \varepsilon^{-1 / 2} \beta^{-5 / 2} \int_{0}^{\frac{1}{2}} x \Phi_{x}(t, x) H_{t}(\alpha, x) \exp \left(-\frac{x^{2}}{4 \varepsilon \beta}\right) \mathrm{d} x \\
& +\varepsilon^{-1 / 2} \beta^{-3 / 2} \frac{1}{4 \varepsilon \beta^{2}} \int_{0}^{\frac{1}{2}} x^{3} \Phi_{x}(t, x) H_{t}(\alpha, x) \exp \left(-\frac{x^{2}}{4 \varepsilon \beta}\right) \mathrm{d} x .
\end{aligned}
$$

Similar estimates yield:

$$
\left|\partial_{s_{2}} Q_{4}\left(t, s_{1}, s\right)\right| \lesssim \varepsilon\left\|H_{t x}\right\|_{\infty}\left\|\Phi_{x}\right\|_{\infty} \cdot \frac{1}{t-s}
$$

Therefore:

$$
\begin{aligned}
\int_{s_{2}}^{\tilde{s_{2}}} \int_{\tilde{s_{2}}}^{1}\left|\partial_{s_{2}} Q_{4}\left(t, s_{1}, s\right)\right| \mathrm{d} t \mathrm{~d} s & \lesssim \varepsilon\left\|H_{t x}\right\|_{\infty}\left\|\Phi_{x}\right\|_{\infty} \cdot \int_{s_{2}}^{\tilde{s_{2}}} \int_{\tilde{s_{2}}}^{1} \frac{\mathrm{d} t \mathrm{~d} s}{t-s} \\
& \lesssim \varepsilon\left\|H_{t x}\right\|_{\infty}\left\|\Phi_{x}\right\|_{\infty} \cdot \int_{s_{2}}^{\tilde{s_{2}}}\left|\ln \left(\tilde{s_{2}}-s\right)\right| \mathrm{d} s \\
& \lesssim \varepsilon\left\|H_{t x}\right\|_{\infty}\left\|\Phi_{x}\right\|_{\infty} \cdot\left|\tilde{s_{2}}-s_{2}\right|\left(1+\ln \left|\tilde{s_{2}}-s_{2}\right|\right)
\end{aligned}
$$

Therefore, for any fixed $p<1$, we have:

$$
\left|R_{4}\left(s_{1}, s_{2}\right)-R_{4}\left(s_{1}, \tilde{s_{2}}\right)\right| \lesssim \varepsilon\left\|H_{t x}\right\|_{\infty}\left\|\Phi_{x}\right\|_{\infty} \cdot\left|\tilde{s_{2}}-s_{2}\right|^{p} .
$$

Thanks to Lemma 19 and Lemma 20, this proves that, for any $\gamma<\frac{1}{16}$,

$$
\kappa\left(\partial_{12} K_{4}\right) \lesssim \exp \left(-\frac{\gamma}{\varepsilon}\right) .
$$

\subsection{Handling the $K_{5}$ kernel}

Recall that $A_{5}$ was defined by:

$$
A_{5}\left(t, s_{1}, s_{2}\right)=\int_{0}^{\frac{1}{2}} \Phi_{x}(1-t, x) H\left(t-s_{2}, x\right) \operatorname{erf}\left(\frac{x}{\sqrt{4 \varepsilon\left(t-s_{1}\right)}}\right) \mathrm{d} x .
$$

First part. The first order derivative $T_{5}$ is null. Indeed,

$$
\begin{aligned}
T_{5}\left(s_{1}, s_{2}\right) & =\left.\left(\partial_{s_{1}} A_{5}\right)\right|_{t=s_{2}} \\
& =\frac{1}{2 \sqrt{\pi \varepsilon}} \int_{0}^{\frac{1}{2}} \Phi_{x}\left(1-s_{2}, x\right) H(0, x) \cdot \frac{x}{\left(s_{2}-s_{1}\right)^{\frac{3}{2}}} \exp \left(-\frac{x^{2}}{4 \varepsilon\left(s_{2}-s_{1}\right)}\right) \mathrm{d} x=0 .
\end{aligned}
$$

Second part. We consider the second order derivative:

$$
Q_{5}\left(t, s_{1}, s_{2}\right)=\partial_{s_{2}} \partial_{s_{1}} A_{5}\left(t, s_{1}, s_{2}\right) \approx \varepsilon^{-1 / 2} \alpha^{-3 / 2} \int_{0}^{\frac{1}{2}} x \Phi_{x}(t, x) H_{t}(\beta, x) \exp \left(-\frac{x^{2}}{4 \varepsilon \alpha}\right) \mathrm{d} x .
$$

Since $H_{t}(t, 0) \equiv 0,\left|H_{t}(t, x)\right| \leq x\left\|H_{t x}\right\|_{\infty}$. Using Lemma 21, we obtain:

$$
\begin{aligned}
\left|Q_{5}\left(t, s_{1}, s_{2}\right)\right| & \lesssim \varepsilon^{-1 / 2} \alpha^{-3 / 2}\left\|H_{t x}\right\|_{\infty}\left\|\Phi_{x}\right\|_{\infty} \int_{0}^{\frac{1}{2}} x^{2} \exp \left(-\frac{x^{2}}{4 \varepsilon \alpha}\right) \mathrm{d} x \\
& \lesssim \varepsilon\left\|H_{t x}\right\|_{\infty}\left\|\Phi_{x}\right\|_{\infty} .
\end{aligned}
$$

By integration over $t \in\left(s_{2}, 1\right)$, we obtain:

$$
\left|R_{5}\left(s_{1}, s_{2}\right)\right| \lesssim \varepsilon\left\|H_{t x}\right\|_{\infty}\left\|\Phi_{x}\right\|_{\infty} .
$$

Differentiating (5.81) with respect to $\alpha$ and proceeding likewise yields:

$$
\left|\partial_{s_{1}} Q_{5}\left(t, s_{1}, s_{2}\right)\right| \lesssim \varepsilon\left\|H_{t x}\right\|_{\infty}\left\|\Phi_{x}\right\|_{\infty} \cdot \frac{1}{\alpha} .
$$


Thus,

$$
\left|R_{5}\left(s_{1}, s_{2}\right)-R_{5}\left(\tilde{s_{1}}, s_{2}\right)\right| \lesssim \varepsilon\left\|H_{t x}\right\|_{\infty}\left\|\Phi_{x}\right\|_{\infty} \cdot \Delta^{-1}\left|\tilde{s_{1}}-s_{1}\right| .
$$

Differentiation with respect to $\beta$ is even easier and gives:

$$
\left|\partial_{s_{2}} Q_{5}\left(t, s_{1}, s_{2}\right)\right| \lesssim \varepsilon\left\|H_{t t x}\right\|_{\infty}\left\|\Phi_{x}\right\|_{\infty},
$$

from which we easily conclude that $R_{5}$ is Lipschitz with respect to $s_{2}$.

Thanks to Lemma 19 and Lemma 20, this proves that, for any $\gamma<\frac{1}{16}$,

$$
\kappa\left(\partial_{12} K_{5}\right) \lesssim \exp \left(-\frac{\gamma}{\varepsilon}\right)
$$

\subsection{Handling the $K_{6}$ kernel}

Recall that $A_{6}$ was defined by:

$$
A_{6}\left(t, s_{1}, s_{2}\right)=\int_{0}^{\frac{1}{2}} \Phi_{x}(1-t, x) H\left(t-s_{1}, x\right) H\left(t-s_{2}, x\right) \mathrm{d} x .
$$

First part. The first order derivative $T_{6}$ is null. Indeed:

$$
T_{6}\left(s_{1}, s_{2}\right)=\left.\left(\partial_{s_{1}} A_{6}\right)\right|_{t=s_{2}}=\int_{0}^{\frac{1}{2}} \Phi_{x}(0, x) H_{t}\left(s_{2}-s_{1}, x\right) H(0, x) \mathrm{d} x=0 .
$$

Second part. We consider the second order derivative:

$$
Q_{6}\left(t, s_{1}, s_{2}\right)=\partial_{s_{2}} \partial_{s_{1}} A_{6}\left(t, s_{1}, s_{2}\right)=\int_{0}^{\frac{1}{2}} \Phi_{x}\left(1-s_{2}, x\right) H_{t}\left(t-s_{1}, x\right) H_{t}\left(t-s_{2}, x\right) \mathrm{d} x
$$

For any $t \in(0,1)$, we estimate:

$$
\begin{aligned}
\left|Q_{6}\left(t, s_{1}, s_{2}\right)\right| \leq & \left\|\Phi_{x}\right\|_{\infty}\left\|H_{t}\right\|_{\infty}^{2} \\
\left|Q_{6}\left(t, s_{1}, s_{2}\right)-Q_{6}\left(t, \tilde{s}_{1}, s_{2}\right)\right| \leq & \left|s_{1}-\tilde{s}_{1}\right| \cdot\left\|\Phi_{x}\right\|_{\infty}\left\|H_{t t}\right\|_{\infty}\left\|H_{t}\right\|_{\infty}, \\
\left|Q_{6}\left(t, s_{1}, s_{2}\right)-Q_{6}\left(t, s_{1}, \tilde{s}_{2}\right)\right| \leq & \left|s_{2}-\tilde{s}_{2}\right| \cdot\left\|\Phi_{x}\right\|_{\infty}\left\|H_{t}\right\|_{\infty}\left\|H_{t t}\right\|_{\infty} \\
& +\left|s_{2}-\tilde{s}_{2}\right| \cdot\left\|\Phi_{t x}\right\|_{\infty}\left\|H_{t}\right\|_{\infty}^{2} .
\end{aligned}
$$

Hence, we can extend these estimates to:

$$
R_{6}\left(s_{1}, s_{2}\right)=\int_{s_{2}}^{1} Q_{6}\left(t, s_{1}, s_{2}\right) \mathrm{d} t
$$

The only non immediate extension is:

$$
\begin{aligned}
\left|R_{6}\left(s_{1}, s_{2}\right)-R_{6}\left(s_{1}, \tilde{s}_{2}\right)\right| \leq & \int_{s_{2}}^{1}\left|Q_{6}\left(t, s_{1}, s_{2}\right)-Q_{6}\left(t, s_{1}, \tilde{s}_{2}\right)\right| \mathrm{d} t+\int_{s_{2}}^{\tilde{s}_{2}}\left|Q_{6}\left(t, s_{1}, \tilde{s}_{2}\right)\right| \mathrm{d} t \\
\leq & \left|s_{2}-\tilde{s}_{2}\right|\left(\left\|\Phi_{x}\right\|_{\infty}\left\|H_{t}\right\|_{\infty}\left\|H_{t t}\right\|_{\infty}\right. \\
& \left.+\left\|\Phi_{t x}\right\|_{\infty}\left\|H_{t}\right\|_{\infty}^{2}+\left\|\Phi_{x}\right\|_{\infty}\left\|H_{t}\right\|_{\infty}^{2}\right)
\end{aligned}
$$

Thanks to Lemma 19 and Lemma 20, this proves that, for any $\gamma<\frac{1}{16}$,

$$
\kappa\left(\partial_{12} K_{6}\right) \lesssim \exp \left(-\frac{\gamma}{\varepsilon}\right)
$$

\subsection{Conclusion of the expansion of $K^{\varepsilon}$}

Lemma 23. There exists $\varepsilon_{1}>0$ and $k_{1}>0$ such that, for any $0<\varepsilon \leq \varepsilon_{1}$ and any $u \in L^{2}(0,1)$,

$$
\left\langle K^{\varepsilon} u, u\right\rangle \geq k_{1} \sqrt{\varepsilon}|U|_{H^{-1 / 4}}^{2} .
$$

Proof. Thanks to the previous paragraphs, we have shown that $K^{\varepsilon}=\frac{\sqrt{\varepsilon}}{45 \sqrt{\pi}} K^{0}+R$, where $R=$ $\tilde{K}_{1}+K_{2}+K_{3}+K_{4}+K_{5}+K_{6}$ is such that $\kappa\left(\partial_{12} R\right) \lesssim \varepsilon^{3 / 2}$. From Lemma 18, we deduce that there exists $C_{0}$ such that, for any $u \in L^{2}(0,1),|\langle R u, u\rangle| \leq C_{0} \varepsilon^{3 / 2}|U|_{H^{-1 / 4}}^{2}$. Moreover, thanks to Lemma 12, there exists $c_{0}$ such that $\left\langle K^{0} u, u\right\rangle \geq c_{0}|U|_{H^{-1 / 4}}^{2}$. Hence, for any $k_{1}<c_{0} /(45 \sqrt{\pi})$, equation (5.94) holds for $\varepsilon$ small enough.

Equation (5.94) gives a very weak coercivity, both because the norm involved is a very weak $H^{-5 / 4}$ norm on the control $u$, and because the coercivity constant $k_{1} \sqrt{\varepsilon}$ decays when $\varepsilon \rightarrow 0$. However, this is enough to overcome the remaining higher order residues, as we prove in the following section. 


\section{Back to the full Burgers non-linear system}

In the first part of this work, we studied a second order approximation of our initial Burgers' system. Thanks to the careful study of an integral kernel, we proved that the projection $\langle\rho, b\rangle$ of the state is coercive with respect to the control $u$, for a given norm. Now, we want to prove that the same fact holds true for the full non-linear system, ie. for the projection $\langle\rho, y\rangle$. In order to do this, we need to provide estimates showing that the projections of the higher order terms in the expansion of the state are smaller than the coercive quantity obtained above. Therefore, we need to prove estimates of $a, b$ and the higher order residues involving the weak $|u|_{H^{-5 / 4}}$ norm.

\subsection{Preliminary estimates on $a$ and $b$}

\subsubsection{Estimating the first order term $a$}

In order to compute $a$ (defined by system (1.8)), a natural idea is to introduce $U$ the primitive of $u$ such that $U(0)=0$. Neglecting the impact of the boundary Dirichlet conditions gives the approximation $a(t, x) \approx U(t)$. To make this exact, we introduce $\tilde{a}$ which is the solution to:

$$
\left\{\begin{aligned}
\tilde{a}_{t}-\varepsilon \tilde{a}_{x x} & =0 & & \text { in }(0,1) \times(0,1), \\
\tilde{a}(t, 0) & =-U(t) & & \text { in }(0,1), \\
\tilde{a}(t, 1) & =-U(t) & & \text { in }(0,1), \\
\tilde{a}(0, x) & =0 & & \text { in }(0,1) .
\end{aligned}\right.
$$

Hence, $a(t, x)=U(t)+\tilde{a}(t, x)$, without any approximation. This decomposition is useful because we write $a$ as the sum of a term which does not depend on $x$ (thus, $a_{x}=\tilde{a}_{x}$ ) and a term whose size is controlled by the desired quantity $|U|_{H^{-1 / 4}}$. Indeed,

Lemma 24. The following estimates hold:

$$
\begin{aligned}
\|\tilde{a}\|_{2} & \lesssim|U|_{H^{-1 / 4}}, \\
\|a\|_{\infty}+\|\tilde{a}\|_{\infty} & \lesssim|u|_{2}, \\
\varepsilon\left\|a_{x}\right\|_{L^{2}\left(L^{\infty}\right)} & \lesssim|u|_{2} .
\end{aligned}
$$

Proof. The first inequality (6.2) is a direct application of estimate (2.16) from Lemma 5.

The second inequality is a consequence of the maximum principle. Indeed, thanks to equation (6.1), $\|\tilde{a}\|_{\infty}$ is smaller than $|U|_{\infty}$. Since $a=U+\tilde{a},\|a\|_{\infty}$ is smaller than $2|U|_{\infty}$. Estimate (6.3) follows because $|U|_{\infty} \leq|u|_{2}$.

The third inequality stems from Lemma 3 . Since $a$ is even, $a_{x}(\cdot, 1 / 2) \equiv 0$. Thus:

$$
\begin{aligned}
\left\|a_{x}\right\|_{L^{2}\left(L^{\infty}\right)}^{2} & =\int_{0}^{1}\left(\sup _{x \in(0,1)}\left|a_{x}(t, x)\right|\right)^{2} \mathrm{~d} t \\
& =\int_{0}^{1}\left(\sup _{x \in(0,1)}\left|\int_{\frac{1}{2}}^{x} a_{x x}\left(t, x^{\prime}\right) \mathrm{d} x^{\prime}\right|\right)^{2} \mathrm{~d} t \\
& \leq \int_{0}^{1} \int_{0}^{1} a_{x x}^{2}\left(t, x^{\prime}\right) \mathrm{d} x^{\prime} \mathrm{d} t .
\end{aligned}
$$

Combined with (2.7), this proves (6.4).

\subsubsection{Estimating the second order term $b$}

Lemma 25. The following estimate holds:

$$
\begin{aligned}
\varepsilon^{1 / 2}\|b\|_{L^{\infty}\left(L^{2}\right)}+\varepsilon\left\|b_{x}\right\|_{L^{2}} & \lesssim|u|_{L^{2}} \cdot|U|_{H^{-1 / 4}} \\
\varepsilon^{3 / 2}\|b\|_{\infty} & \lesssim|u|_{2}^{2} \\
\varepsilon^{3 / 2}\left\|b_{x}\right\|_{L^{2}\left(L^{\infty}\right)} & \lesssim|u|_{2}^{2}
\end{aligned}
$$


Proof. For the first inequality, we want to apply Lemma 6 . Hence, we want to write the source term in equation (1.9) as a spatial derivative. Writing $-a a_{x}=-\partial_{x}\left(a^{2} / 2\right)$ would not lead to the required estimates. In order for the weak $H^{-1 / 4}$ norm to appear, we need to introduce $\tilde{a}$. Indeed, using the decomposition $a(t, x)=U(t)+\tilde{a}(t, x)$, we can write:

$$
-a a_{x}=-a \tilde{a}_{x}=-\frac{\mathrm{d}}{\mathrm{d} x}\left[a \tilde{a}-\frac{1}{2} \tilde{a}^{2}\right] .
$$

The term under the derivative can easily be estimated in $L^{2}$ :

$$
\left\|a \tilde{a}-\frac{1}{2} \tilde{a}^{2}\right\|_{L^{2}} \leq\|\tilde{a}\|_{L^{2}} \cdot\left(\|a\|_{\infty}+\|\tilde{a}\|_{\infty}\right) \lesssim|u|_{L^{2}} \cdot|U|_{H^{-1 / 4}},
$$

where the last inequality comes from Lemma 24. Thus, we can apply Lemma 6 to prove (6.6).

For the second and third inequalities, thanks to Lemma $3,\left\|a_{x}\right\|_{2} \lesssim \varepsilon^{-1 / 2}|u|_{2}$. Moreover, thanks to Lemma $24,\|a\|_{\infty} \lesssim|u|_{2}$. Thus, $\left\|a a_{x}\right\|_{2} \lesssim \varepsilon^{-1 / 2}|u|_{2}^{2}$. We can apply Lemma 3 to show that $\|b\|_{X_{1}} \lesssim \varepsilon^{-3 / 2}|u|_{2}^{2}$. Inequality (6.7) follows from the injection $X_{1} \hookrightarrow L^{\infty}$ (see (2.4) from Lemma 1).

Moreover, since $\int_{0}^{1} b_{x}(t, x) \mathrm{d} x=b(t, 1)-b(t, 0)=0$ for any $t \in(0,1)$, the mean value of $b_{x}(t, \cdot)$ is 0 . Thus, $\left|b_{x}(t, \cdot)\right|_{\infty} \leq\left|b_{x x}(t, \cdot)\right|_{2}$. Hence, $\left\|b_{x}\right\|_{L^{2}\left(L^{\infty}\right)} \leq\left\|b_{x x}\right\|_{2}$. This proves estimate (6.8).

\subsection{Non-linear residue}

Let us expand $y$ as $a+b+r$, where $a$ stands for the first order linear approximation, $b$ stands for the second quadratic order and $r$ is a (small) residue. Therefore, $r$ is the solution to:

$$
\left\{\begin{aligned}
r_{t}-\varepsilon r_{x x} & =-r r_{x}-[(a+b) r]_{x}-\left[a b+\frac{1}{2} b^{2}\right]_{x} & & \text { in }(0,1) \times(0,1), \\
r(t, 0) & =0 & & \text { in }(0,1), \\
r(t, 1) & =0 & & \text { in }(0,1), \\
r(0, x) & =0 & & \text { in }(0,1) .
\end{aligned}\right.
$$

Lemma 26. System (6.11) admits a unique solution $r \in X_{1}$. Moreover, under the assumption:

$$
|u|_{2} \leq \varepsilon^{3 / 2}
$$

the following estimate holds:

$$
\|r\|_{2}+\left\|r_{t}\right\|_{2} \lesssim \varepsilon^{-3 / 2}|u|_{2}^{2}|U|_{H^{-1 / 4}}
$$

Proof. The existence of $r \in X_{1}$ can be deduced directly from the equality $r=y-a-b$. To prove the estimate, we will use Lemma 7 with a null initial data, $w=-(a+b)$ and $g=-a b-\frac{1}{2} b^{2}$. To apply estimate (2.20), we start by computing the norms of $w$ and $g$ that we need. We start with $w=-(a+b)$. Combining (6.3), (6.7) and (6.12) gives:

$$
\|w\|_{\infty} \leq\|a\|_{\infty}+\|b\|_{\infty} \lesssim|u|_{2}+\varepsilon^{-3 / 2}|u|_{2}^{2} \lesssim|u|_{2}
$$

In particular, (6.14) and (6.12) yield:

$$
\gamma=\frac{1}{\varepsilon}\|w\|_{L^{2}\left(L^{\infty}\right)}^{2} \leq \frac{1}{\varepsilon}\|w\|_{\infty}^{2} \leq \frac{1}{\varepsilon}|u|_{2}^{2} \lesssim 1
$$

Finally, combining (6.4) and (6.8):

$$
\left\|w_{x}\right\|_{L^{2}\left(L^{\infty}\right)} \leq\left\|a_{x}\right\|_{L^{2}\left(L^{\infty}\right)}+\left\|b_{x}\right\|_{L^{2}\left(L^{\infty}\right)} \lesssim \varepsilon^{-1}|u|_{2}+\varepsilon^{-3 / 2}|u|_{2}^{2} \lesssim \varepsilon^{-1}|u|_{2} .
$$

We move on to $g=-a b-\frac{1}{2} b^{2}$. Combining (6.3), (6.6), (6.7) and (6.12) gives:

$$
\begin{aligned}
\|g\|_{2} & \leq\left(\|a\|_{\infty}+\|b\|_{\infty}\right)\|b\|_{2} \\
& \leq\left(|u|_{2}+\varepsilon^{-3 / 2}|u|_{2}^{2}\right) \varepsilon^{-1 / 2}|u|_{2}|U|_{H^{-1 / 4}} \\
& \leq \varepsilon^{-1 / 2}|u|_{2}^{2}|U|_{H^{-1 / 4}}
\end{aligned}
$$


Combining (6.3), (6.6), (6.7) and (6.12), we obtain:

$$
\begin{aligned}
\|g\|_{L^{2}\left(L^{\infty}\right)} & \leq\left(\|a\|_{\infty}+\|b\|_{\infty}\right) \cdot\|b\|_{L^{2}\left(L^{\infty}\right)} \\
& \leq\left(\|a\|_{\infty}+\|b\|_{\infty}\right) \cdot\left\|b_{x}\right\|_{2} \\
& \lesssim \varepsilon^{-1}|u|_{2}^{2}|U|_{H^{-1 / 4}} .
\end{aligned}
$$

Lastly, mixing (6.3), (6.4), (6.6), (6.7) and (6.12) gives:

$$
\begin{aligned}
\left\|g_{x}\right\|_{2} & \leq\left\|a_{x}\right\|_{L^{2}\left(L^{\infty}\right)}\|b\|_{L^{\infty}\left(L^{2}\right)}+\|a\|_{\infty}\left\|b_{x}\right\|_{2}+\|b\|_{\infty}\left\|b_{x}\right\|_{2} \\
& \lesssim \varepsilon^{-3 / 2}|u|_{2}^{2}|U|_{H^{-1 / 4}}+\varepsilon^{-1}|u|_{2}^{2}|U|_{H^{-1 / 4}}+\varepsilon^{-5 / 2}|u|_{2}^{3}|U|_{H^{-1 / 4}} \\
& \lesssim \varepsilon^{-3 / 2}|u|_{2}^{2}|U|_{H^{-1 / 4}} .
\end{aligned}
$$

Eventually, plugging estimates (6.14)-(6.19) into the main estimation (2.20), yields:

$$
\left\|r_{t}\right\|_{2} \lesssim \varepsilon^{-3 / 2}|u|_{2}^{2}|U|_{H^{-1 / 4}} .
$$

From (6.20) and the initial condition $r(0, \cdot)=0$, we conclude (6.13).

Lemma 27. Under the assumption (6.12), we have:

$$
|\langle\rho, r(1, \cdot)\rangle| \lesssim \varepsilon^{-3 / 2}|u|_{2}^{2}|U|_{H^{-1 / 4}}^{2} .
$$

Proof. This lemma is not a direct consequence of Lemma 26. Indeed, estimate (6.13) only involves $|U|_{H^{-1 / 4}}$ with an exponent of 1 . To obtain estimate (6.21), we need to work a little more. Using Lemma 10 and equation (6.11), we can compute:

$$
\begin{aligned}
\langle\rho, r(1, \cdot)\rangle & =\int_{0}^{1} \int_{0}^{1} \Phi_{x}\left[a b+\frac{1}{2} b^{2}+(a+b) r+\frac{1}{2} r^{2}\right] \\
& =\int_{0}^{1} \int_{0}^{1} \Phi_{x}(1-t, x) U(t) r(t, x) \mathrm{d} x \mathrm{~d} t+\int_{0}^{1} \int_{0}^{1} \Phi_{x}\left[\frac{1}{2} b^{2}+(\tilde{a}+b) r+\frac{1}{2} r^{2}\right] .
\end{aligned}
$$

We used the fact that $a=U+\tilde{a}$ and the fact that $\Phi_{x} a b$ is an odd function, whose space integral is thus null. The second term is easy to estimate, because we know how to estimate $\tilde{a}, b$ and $r$ in $L^{2}$ using $|U|_{H^{-1 / 4}}$. Thus, we know it will be smaller than $|U|_{H^{-1 / 4}}^{2}$. The first term needs more care.

$$
\int_{0}^{1} U(t) \int_{0}^{1} \Phi_{x}(1-t, x) r(t, x) \mathrm{d} x \mathrm{~d} t=\langle U, v\rangle_{H^{-1}, H_{0}^{1}}
$$

where we introduce $v(t)=\int_{0}^{1} \Phi_{x}(t, x) r(t, x) \mathrm{d} x$ for $t \in(0,1)$. Since $\Phi(0, \cdot) \equiv 0$ and $r(0, \cdot) \equiv 0$, $v(0)=v(1)=0$. Now we compute its $H_{0}^{1}$ norm:

$$
\begin{aligned}
\int_{0}^{1} v_{t}(t)^{2} \mathrm{~d} t & =\int_{0}^{1}\left(\int_{0}^{1} \Phi_{t x}(1-t, x) r(t, x)+\Phi_{x}(1-t, x) r_{t}(t, x) \mathrm{d} x\right)^{2} \mathrm{~d} t \\
& \leq 2 \int_{0}^{1} \int_{0}^{1} \Phi_{t x}^{2} r^{2}+\Phi_{x}^{2} r_{t}^{2} \\
& \leq 2\left(\left\|\Phi_{t x}\right\|_{\infty}^{2}\|r\|_{2}^{2}+\left\|\Phi_{x}\right\|_{\infty}^{2}\left\|r_{t}\right\|_{2}^{2}\right) \\
& \lesssim \varepsilon^{2}\|r\|_{2}^{2}+\left\|r_{t}\right\|_{2}^{2} \\
& \lesssim\left\|r_{t}\right\|_{2}^{2},
\end{aligned}
$$

where we used estimates (5.19) and (5.21) to estimate $\Phi$. Let us finish the proof.

$$
\begin{aligned}
|\langle\rho, r(1, \cdot)\rangle| & \leq\left|\langle U, v\rangle_{H^{-1}, H_{0}^{1}}\right|+\left|\int_{0}^{1} \int_{0}^{1} \Phi_{x}\left(\frac{1}{2} b^{2}+(\tilde{a}+b) r+\frac{1}{2} r^{2}\right)\right| \\
& \lesssim|U|_{H^{-1}}\left\|r_{t}\right\|_{2}+\left\|\Phi_{x}\right\|_{\infty}\left(\|b\|_{2}^{2}+\|\tilde{a}\|_{2}\|r\|_{2}+\|r\|_{2}^{2}\right) \quad \text { using (6.22) and (6.23), }
\end{aligned}
$$

From (5.19), we know that $\left\|\Phi_{x}\right\|_{\infty} \lesssim 1$. Moreover, $|U|_{H^{-1}} \lesssim|U|_{H^{-1 / 4}}$. Thanks to (6.2), (6.6), (6.13) and (6.12), we conclude from $(6.25)$ that $|\langle\rho, r(1, \cdot)\rangle| \lesssim \varepsilon^{-3 / 2}|u|_{2}^{2}|U|_{H^{-1 / 4}}^{2}$. This concludes the proof of Lemma 27. 


\subsection{A first drifting result concerning reachability from zero}

The null reachability problem consists in computing the set of states that can be reached in time $T$, starting from $y(0, x) \equiv 0$ using a control $u$. Of course, when dealing with viscous equations like (1.1), one may only hope to reach sufficiently smooth states. Theorem 2 tells us that, if the time $T$ is too small, we can never reach a state $y^{1}(x)$ in time $T$ if $\left\langle\rho, y^{1}\right\rangle<0$, whatever the control $u$ (and the smoothness of $\left.y^{1}\right)$. In some sense, the state drifts towards the direction $+\rho$, as a result of the action of the control.

Theorem 2. There exist $T_{2}, k_{2}>0$ such that, for any $0<T<T_{2}$ and any $u \in L^{2}(0, T)$ such that $|u|_{L^{2}(0, T)} \leq 1$, the solution $y \in X_{T}$ to system (1.1) starting from the null initial condition $y(0, x) \equiv 0$ satisfies:

$$
\langle\rho, y(T, \cdot)\rangle \geq k_{2}|U|_{H^{-1 / 4}(0, T)}^{2},
$$

where $U$, as above, is the primitive of $u$ such that $U(0)=0$.

Proof. We are going to use the scaling argument introduced in paragraph 1.4. Thus, from now on, we reintroduce the tilda signs for functions defined on the scaled time interval $(0,1)$. From Lemma 23, we know that, for $\varepsilon<\varepsilon_{1},\left\langle K^{\varepsilon} \tilde{u}, \tilde{u}\right\rangle \geq k_{1} \sqrt{\varepsilon}|\tilde{U}|_{H^{-1 / 4}}^{2}$. From Lemma 27, we know that there exists $c_{2}$ such that, as soon as $|\tilde{u}|_{2} \leq \varepsilon^{3 / 2},|\langle\rho, r(1, \cdot)\rangle| \leq c_{2} \varepsilon^{3 / 2}|\tilde{U}|_{H^{-1 / 4}}^{2}$. Hence, if we consider $\tilde{y}$ the solution to (1.7), write $\tilde{y}=a+b+r$, for any $0<k_{2}<k_{1}$, there exists $\varepsilon_{2}>0$ such that, for $\varepsilon<\varepsilon_{2}$, $\langle\rho, \tilde{y}(1, \cdot)\rangle \geq k_{2} \sqrt{\varepsilon}|\tilde{U}|_{H^{-1 / 4}}^{2}$. Recalling that $\tilde{u}(t)=\varepsilon^{2} u(\varepsilon t)$ and $\tilde{y}(t, x)=\varepsilon y(\varepsilon t, x)$, we obtain:

$$
\langle\rho, y(\varepsilon, \cdot)\rangle=\left\langle\frac{1}{\varepsilon} \tilde{y}(1, \cdot), \rho\right\rangle \geq k_{2} \varepsilon^{-1 / 2}|\tilde{U}|_{H^{-1 / 4}(0,1)}^{2} \geq k_{2}|U|_{H^{-1 / 4}(0, \varepsilon)}^{2},
$$

under the assumption:

$$
|\tilde{u}|_{L^{2}(0,1)} \leq \varepsilon^{3 / 2} \Leftrightarrow|u|_{L^{2}(0, \varepsilon)} \leq 1 .
$$

Theorem 2 follows from (6.27) and (6.28) with $T_{2}=\varepsilon_{2}$. Equation (6.28) is obtained via a direct change of variable. To establish (6.27), one can compute the weak $H^{-1 / 4}$ norms using Fourier transforms.

\subsection{Persistance of projections in absence of control}

We start by remarking that, when no control is used, the projection of the state against any fixed profile $\mu \in L^{2}(0,1)$ remains almost constant in small time.

Lemma 28. Let $T>0, \mu \in L^{2}(0,1)$ and $y^{0} \in H_{0}^{1}(0,1) \cap H^{2}(0,1)$. Assume that $\left|y^{0}\right|_{H^{2}} \leq 1$. Consider $y \in X_{T}$ the solution to system (1.1) with initial data $y^{0}$ and null control $(u=0)$. Then,

$$
\langle\mu, y(T, \cdot)\rangle=\left\langle\mu, y^{0}\right\rangle+\mathcal{O}\left(T^{1 / 2}|\mu|_{2}\left|y^{0}\right|_{H^{2}}\right)
$$

Proof. We decompose $y=y^{0}+z$. Hence, $z$ is the solution to:

$$
\left\{\begin{aligned}
z_{t}-z_{x x}+z z_{x} & =\left(y^{0} z\right)_{x}+y_{x x}^{0}-y^{0} y_{x}^{0} & & \text { in }(0, T) \times(0,1), \\
z(t, 0) & =0 & & \text { in }(0, T), \\
z(t, 1) & =0 & & \text { in }(0, T), \\
z(0, x) & =0 & & \text { in }(0,1) .
\end{aligned}\right.
$$

Thus, we can apply Lemma 7 with $w(t, x)=y^{0}(x)$ and $g(t, x)=y_{x}^{0}-\frac{1}{2}\left(y^{0}\right)^{2}$ to system (6.30). Estimate (2.20) tells us that $\left\|z_{t}\right\|_{2} \lesssim\left|y^{0}\right|_{H^{2}}$. Here, we need the assumption that $\left|y^{0}\right|_{H^{2}} \leq C$, where $C$ is any fixed constant, in order to avoid propagating non-linear estimates (involving exponentials). Since $z(0, x) \equiv 0$, we can write:

$$
|\langle\mu, z(T, \cdot)\rangle|=\left|\int_{0}^{T} \int_{0}^{1} z_{t} \mu\right| \leq T^{1 / 2}\left\|z_{t}\right\|_{2}|\mu|_{2} .
$$

The conclusion (6.29) follows from (6.31). 


\subsection{Proof of Theorem 1}

Let us finish the proof of Theorem 1. We consider an initial data of the form $y^{\delta}=\delta \rho$, where $\delta>0$ can be picked as small as we need and $\rho$ is defined in (3.14). Please note that many other initial data cannot be driven back to zero in short time with small controls. However, to prove Theorem 1, it is sufficient to exhibit a single sequence.

For $T>0, u \in L^{2}(0, T)$ and $\delta>0$, we consider $y \in X_{T}$, the solution to system (1.1) with initial data $y^{\delta}$ and control $u$. To isolate the different contributions, we decompose $y$ as $\bar{y}+y^{u}+z$, where:

$$
\begin{gathered}
\left\{\begin{array} { r l } 
{ \overline { y } _ { t } - \overline { y } _ { x x } + \overline { y } \overline { y } _ { x } = 0 } & { \text { in } ( 0 , T ) \times ( 0 , 1 ) , } \\
{ \overline { y } ( t , 0 ) = 0 } & { \text { in } ( 0 , T ) , } \\
{ \overline { y } ( t , 1 ) = 0 } & { \text { in } ( 0 , T ) , } \\
{ \overline { y } ( 0 , x ) = y ^ { \delta } } & { \text { in } ( 0 , 1 ) , }
\end{array} \left\{\begin{array}{rl}
y_{t}^{u}-y_{x x}^{u}+y^{u} y_{x}^{u}=u(t) & \text { in }(0, T) \times(0,1), \\
y^{u}(t, 0)=0 & \text { in }(0, T), \\
y^{u}(t, 1)=0 & \text { in }(0, T), \\
y^{u}(0, x)=0 & \text { in }(0,1),
\end{array}\right.\right. \\
\left\{\begin{aligned}
z_{t}-z_{x x}+z z_{x}=-\left[\left(\bar{y}+y^{u}\right) z\right]_{x}-\left[\bar{y} y^{u}\right]_{x} & \text { in }(0, T) \times(0,1), \\
z(t, 0)=0 & \text { in }(0, T), \\
z(t, 1)=0 & \text { in }(0, T), \\
z(0, x)=0 & \text { in }(0,1) .
\end{aligned}\right.
\end{gathered}
$$

Hence, $\bar{y}$ captures the free movement starting from the initial data $y^{\delta}$ while $y^{u}$ corresponds to the action of the control starting from a null initial data. Systems (6.32)-(6.34) allow us to decouple these two contributions. The term $z$ is a small residue with homogeneous boundary and initial data.

First, let us apply Lemma 8 to system (6.32). Estimates (2.28) and (2.29) yield:

$$
\begin{aligned}
\left\|\bar{y}_{x x}\right\|_{2}+\left\|\bar{y}_{x}\right\|_{2}+\left\|\bar{y}_{t}\right\|_{2} & \lesssim \delta, \\
\|\bar{y}\|_{\infty} & \leq\left|y^{0}\right|_{\infty} \lesssim \delta .
\end{aligned}
$$

Similarly, we apply Lemma 8 to system (6.33). If we assume that $|u|_{2} \leq 1$ and $T \leq 1$, we obtain:

$$
\begin{aligned}
\left\|y_{x x}^{u}\right\|_{2}+\left\|y_{x}^{u}\right\|_{2}+\left\|y_{t}^{u}\right\|_{2} & \lesssim|u|_{2}, \\
\left\|y^{u}\right\|_{\infty} & \leq|u|_{2} .
\end{aligned}
$$

Next, we look at system (6.34). We apply Lemma 7 with $w=-\left(\bar{y}+y^{u}\right), g=-\bar{y} y^{u}$ and a null initial data. Combining (6.35) and (6.36) yields the necessary estimates:

$$
\begin{aligned}
\|g\|_{2}+\left\|g_{x}\right\|_{2}+\|g\|_{L^{2}\left(L^{\infty}\right)} & \lesssim \delta|u|_{2}, \\
\|w\|_{\infty}+\|w\|_{L^{2}\left(L^{\infty}\right)}\|w\|_{L^{2}\left(L^{\infty}\right)} & \lesssim \delta+|u|_{2} .
\end{aligned}
$$

Hence, (6.38) yields $\gamma \lesssim 1$. Therefore, plugging (6.37) and (6.38) into (2.20) gives:

$$
\left\|z_{x x}\right\|_{2}+\left\|z_{t}\right\|_{2} \lesssim \delta|u|_{2} .
$$

Once again, we use the initial condition $z(0, \cdot) \equiv 0$ and (6.39) to compute:

$$
|\langle\rho, z(T, \cdot)\rangle|=\left|\int_{0}^{T} \int_{0}^{1} z_{t} \rho\right| \lesssim T^{1 / 2} \delta|u|_{2} .
$$

Now, assuming $T \leq T_{2}$, we can combine Theorem 2 and Lemma 28 with (6.40) to obtain:

$$
\langle y(T, \cdot), \rho\rangle \geq \delta|\rho|_{2}^{2}+k_{2}|U|_{H^{-1 / 4}}^{2}+\mathcal{O}\left(T^{1 / 2} \delta\left(1+|u|_{2}\right)\right) .
$$

From (6.41), we deduce that $\langle\rho, y(T, \cdot)\rangle>0$ as soon as $T$ is small enough and under the assumption $|u|_{2} \leq 1$. Thus, we have proved Theorem 1 with $\eta=1$. 


\section{Conclusion and perspectives}

We expect that the methodology followed in this paper can be used for a wide variety of non-linear systems involving a single scalar control. Indeed, when studying small time local controllability for some formal system $\dot{y}=F(y, u(t))$, the first step is always to consider the linearized system, $\dot{a}=\partial_{y} F(0) a+\partial_{u} F(0) u$. When this system is controllable, fixed point or inverse mapping theorems often allow us to deduce that the non-linear system is small time locally controllable. When the linearized system is not controllable, we can decompose the state $y$ as $a+b$, where the (linear) component $a$ is controllable and the second component $b$ is indirectly controlled through a quadratic source term involving $a$ (and/or, sometimes, $u$ ).

What our proof demonstrates, is that it is possible, even for infinite dimensional systems, to express projections of the second order part $b$ as kernels acting on the control. The careful study of these kernels can then lead ever to negative results (like it is the case here, because we prove a coercivity lemma), or to positive results (if the kernel is found to have both positive and negative eigenvalues, we can hope to prove that the system can be driven in the two opposite directions).

It is worth to be noted that the coercivity used in this paper, although it involves a weak $H^{-5 / 4}$ norm of the control $u$, is in fact pretty strong. Indeed, it was obtained for any small $u \in L^{2}$. It would have been sufficient to prove the coercivity of the kernel $K^{\varepsilon}$ on the strict subspace:

$$
\mathcal{V}_{\varepsilon}=\left\{u \in L^{2}(0,1), \quad a(t=1, \cdot) \equiv 0, \quad \text { where } a \text { is the solution to system }(1.8)\right\} .
$$

For other systems, it may be easier (or necessary) to restrict the study of the integral operator $K^{\varepsilon}$ to the subspace $\mathcal{V}_{\varepsilon}$ in order to obtain a conclusion.

As a perspective, an example of such an open problem is the small time controllability of the nonlinear Korteweg de Vries equation for critical domains. Indeed, in [41], Rosier proved that the KdV equation was small time locally controllable for non critical domains using the linearized system. Then in [21], Coron and Crépeau proved that, for the first critical length, small time local controllability holds thanks to a third order expansion. In [15] and [16], Cerpa then Cerpa and Crépeau proved that large time local controllability holds for all critical lengths. It remains an open question to know whether small time local controllability holds for the second critical length. Maybe our method could be adapted to this setting or inspire a new proof.

The author thanks Sergio Guerrero for having attracted his attention on this control problem and his advisor Jean-Michel Coron for his support and ideas all along the elaboration of this proof.

\section{A Weakly singular integral operators}

This appendix is devoted to an explanation of Lemma 18. Although a full proof would exceed the scope of this article, we provide here a brief overview of a general method introduced by Torres in [44] to study the regularization properties of weakly singular integral operators. Our presentation is also inspired by a posterior work of Youssfi, who states a very closely related lemma in [47, Remark 6.a].

Let $n \geq 1$. Singular integral operators on $\mathbb{R}^{n}$ have been extensively studied since the seminal works of Calderón and Zygmund (see [14] and [13]). These integral operators are defined by the singularity of their kernel along the diagonal by an estimate of the form:

$$
|K(x, y)| \leq C|x-y|^{-n} .
$$

In estimate (A.1), the exponent $-n$ is critical. Indeed, the margins of such kernels are almost in $L_{\mathrm{loc}}^{1}$. Here, we are interested in a class of integral operators for which the singularity along the diagonal is weaker. Thus, we expect that they exhibit better smoothing properties. Throughout this section, we denote $\Omega=\left\{(x, y) \in \mathbb{R}^{n} \times \mathbb{R}^{n}, x \neq y\right\}$.

Definition 4 (Weakly singular integral operator). Let $0<s<1$ and $0<\delta \leq 1$. Consider a kernel $K$, continuous on $\Omega$, satisfying:

$$
\begin{aligned}
& |K(x, y)| \leq \kappa|x-y|^{-n+s}, \\
& \left|K\left(x^{\prime}, y\right)-K(x, y)\right| \leq \kappa\left|x^{\prime}-x\right|^{\delta}|x-y|^{-n+s-\delta}, \quad \text { for }\left|x^{\prime}-x\right| \leq \frac{1}{2}|x-y| \text {, } \\
& \left|K\left(x, y^{\prime}\right)-K(x, y)\right| \leq \kappa\left|y^{\prime}-y\right|^{\delta}|x-y|^{-n+s-\delta}, \quad \text { for }\left|y^{\prime}-y\right| \leq \frac{1}{2}|x-y| \text {. }
\end{aligned}
$$


We introduce the associated integral operator $T_{K}$, continuous from $\mathcal{D}\left(\mathbb{R}^{n}\right)$ to $\mathcal{D}^{\prime}\left(\mathbb{R}^{n}\right)$, by defining:

$$
\forall f \in \mathcal{D}\left(\mathbb{R}^{n}\right), \forall x \in \mathbb{R}^{n}, T_{K}(f)(x)=\int K(x, y) f(y) \mathrm{d} y
$$

Under these assumptions, we write $T_{K} \in \operatorname{WSIO}(s, \delta)$.

Definition 4 can be extended for $s \geq 1$. Conditions (A.2), (A.3) and (A.4) must then be extended to the derivatives $\partial_{x}^{\alpha} \partial_{y}^{\beta} K$ for $\alpha+\beta \leq s$. We restrict ourselves to the simpler setting $0<s<1$ as it is sufficient for our study. Note that we define the operator $T_{K}$ from its kernel $K$ (as this is the case for our applications). Proceeding the other way around is possible but would require more care in the sequel (namely, the so-called weak boundedness property to ensure that (A.5) holds; see [47]).

\section{A.1 Atomic and molecular decompositions for Triebel-Lizorkin spaces}

We recall the definitions of classical functional spaces involved in this appendix. Let $\varphi \in \mathcal{S}\left(\mathbb{R}^{n}\right)$ be such that $\varphi(\xi)=0$ for $|\xi| \geq 1$ and $\varphi(\xi)=1$ for $|\xi| \leq \frac{1}{2}$. We introduce $\psi(\xi)=\varphi(\xi / 2)-\varphi(\xi)$. Hence, $\psi \in \mathcal{S}\left(\mathbb{R}^{n}\right)$ and is supported in the annulus $\left\{\frac{1}{2} \leq|\xi| \leq 2\right\}$. We will denote $\dot{\Delta}_{j}$ and $\dot{S}_{j}$ the convolution operators with symbols $\psi\left(2^{-j} \xi\right)$ and $\varphi\left(2^{-j} \xi\right)$.

Definition 5 (Homogeneous Besov space). For $\alpha \in \mathbb{R}, 1 \leq p, q \leq \infty$, the homogeneous Besov space $\dot{B}_{p}^{\alpha, q}$ is defined by the finiteness of the norm (with standard modification for $q=\infty$ ):

$$
\|f\|_{\dot{B}_{p}^{\alpha, q}}=\left(\sum_{j \in \mathbb{Z}} 2^{\alpha q j}\left\|\dot{\Delta}_{j} f\right\|_{p}^{q}\right)^{1 / q} .
$$

Definition 6 (Homogeneous Triebel-Lizorkin space). For $\alpha \in \mathbb{R}, 1 \leq p, q<\infty$, the homogeneous Triebel-Lizorkin space $\dot{F}_{p}^{\alpha, q}$ is defined by the finiteness of the norm:

$$
\|f\|_{\dot{F}_{p}^{\alpha, q}}=\left\|\left(\sum_{j \in \mathbb{Z}} 2^{\alpha q j}\left|\dot{\Delta}_{j} f\right|^{q}\right)^{1 / q}\right\|_{p} .
$$

Frazier and Jawerth introduced atoms and molecules both in the context of Besov spaces ([26]) and Triebel-Lizorkin spaces ([27] and [28]). They proved that the norms on these spaces are then translated into sequential norms on the sequence of coefficients of the decomposition. A linear operator will be continuous between two Triebel-Lizorkin spaces if and only if it maps smooth atoms of the first to smooth molecules of the second. The following definitions are borrowed from [44]. For simplicity, we restrict them to the case $1 \leq p, q \leq+\infty$.

Definition 7 (Smooth atom). Let $\alpha \in \mathbb{R}$ and $Q$ be a dyadic cube in $\mathbb{R}^{n}$ of side length $\ell_{Q}$. A smooth $\alpha$-atom, associated with the cube $Q$ is a function $a \in \mathcal{D}\left(\mathbb{R}^{n}\right)$ satisfying:

$$
\begin{gathered}
\operatorname{supp}(a) \subset 3 Q, \\
\int x^{\gamma} a(x) \mathrm{d} x=0, \quad \forall|\gamma| \leq \max \{0,[-\alpha]\}, \\
\left|\partial_{x}^{\gamma} a(x)\right| \leq \ell_{Q}^{-|\gamma|}, \quad \forall|\gamma| \leq \max \{0,[\alpha]\}+1 .
\end{gathered}
$$

In condition (A.8), 3Q denotes the cube with same center as $Q$ but a tripled side length. It is worth to be noted that multiple normalization choices are possible for condition (A.10). We choose to only include the decay corresponding to the smoothness of the atom. This choice only impacts the formula to compute the size of a function from its decomposition on atoms. We have the following representation theorem:

Lemma 29 (Theorem 5.11, [29]). Let $\alpha \in \mathbb{R}, 1 \leq p, q<\infty$. Let $f \in \dot{F}_{p}^{\alpha, q}$. There exists a sequence of reals $\left(s_{Q}\right)_{Q \in \mathcal{Q}}$ indexed by the set $\mathcal{Q}$ of dyadic cubes of $\mathbb{R}^{n}$ and a sequence of atoms $\left(a_{Q}\right)_{Q \in \mathcal{Q}}$ such that $f=\sum_{Q} s_{Q} a_{Q}$. Moreover, there exists a constant $C$ independent on $f$ such that:

$$
\left\|\left(\sum_{Q} \ell_{Q}^{-\alpha q}\left|s_{Q}\right|^{q}\left|\chi_{Q}(x)\right|^{q}\right)^{1 / q}\right\|_{p} \leq C\|f\|_{\dot{F}_{p}^{\alpha, q}} .
$$


The reciprocal inequality to (A.11) is true even for a wider class of functions, the class of molecules.

Definition 8 (Smooth molecule). Let $\alpha \in \mathbb{R}, M>n$ and $\alpha-[\alpha]<\delta \leq 1$. Let $Q$ be a dyadic cube in $\mathbb{R}^{n}$ of side length $\ell_{Q}$ and center $x_{Q} . A(\delta, M)$ smooth $\alpha$-molecule associated with $Q$ is a function $m$ satisfying:

$$
\begin{gathered}
|m(x)| \leq\left(1+\ell_{Q}^{-1}\left|x-x_{Q}\right|\right)^{-\max \{M, M-\alpha\}}, \\
\int x^{\gamma} m(x) \mathrm{d} x=0, \quad \forall|\gamma| \leq[-\alpha] \\
\left|\partial_{x}^{\gamma} m(x)\right| \leq \ell_{Q}^{-|\gamma|}\left(1+\ell_{Q}^{-1}\left|x-x_{Q}\right|\right)^{-M}, \quad \forall|\gamma| \leq[\alpha], \\
\left|\partial_{x}^{\gamma} m(x)-\partial_{x}^{\gamma} m\left(x^{\prime}\right)\right| \leq \ell_{Q}^{-|\gamma|-\delta}\left|x-x^{\prime}\right|^{\delta} \sup _{|z| \leq\left|x-x^{\prime}\right|}\left(1+\ell_{Q}^{-1}\left|z-\left(x-x_{Q}\right)\right|\right)^{-M}, \quad \forall|\gamma|=[\alpha] .
\end{gathered}
$$

In the definition of a molecule, conditions (A.14) and (A.15) are void by convention if $\alpha<0$. When $\alpha \geq 0$, condition (A.14) implies (A.12). When $\alpha>0$, condition (A.13) is void. We have:

Lemma 30 (Theorem 5.18, [29]). Let $\alpha \in \mathbb{R}, M>n$ and $\alpha-[\alpha]<\delta \leq 1$. Consider a sequence of reals $\left(s_{Q}\right)_{Q \in \mathcal{Q}}$ indexed by the set $\mathcal{Q}$ of dyadic cubes of $\mathbb{R}^{n}$ and a sequence of $(\delta, M)$ smooth $\alpha$-molecules $\left(m_{Q}\right)_{Q \in \mathcal{Q}}$. Let $f=\sum_{Q} s_{Q} m_{Q}$. There exists a constant $C$ independent on $f$ such that:

$$
\|f\|_{\dot{F}_{p}^{\alpha, q}} \leq C\left\|\left(\sum_{Q} \ell_{Q}^{-\alpha q}\left|s_{Q}\right|^{q}\left|\chi_{Q}(x)\right|^{q}\right)^{1 / q}\right\|_{p} .
$$

\section{A.2 Circumventing the $T(1)=0$ condition}

When dealing with singular integral operators, difficulties arise when $T(1) \neq 0$. Most regularity results involve some smoothness condition on $T(1)$ (see, for example the early paper [23]). To circumvent this difficulty when handling weakly singular integral operators, we will write $T_{K}=\tilde{T}_{K}+\pi$ where $\tilde{T}_{K}$ satisfies the same regularity estimates as $T_{K}$ but is such that $\tilde{T}_{K}(1)=0$ and $\pi$ is defined as a paraproduct, for which we can get direct smoothing estimates in the appropriate spaces. For two functions $f, g$, we introduce the following paraproduct $\pi$, inspired by ideas of J.-M. Bony (see the seminal work [10], the nice introduction to paraproducts [8] for a quick overview or [3, Section 2.6.1] for a complete detailed presentation):

$$
\pi_{g}(f)=\sum_{j \in \mathbb{Z}} \dot{\Delta}_{j}(g) \dot{S}_{j-2}(f) .
$$

Lemma 31 (Lemma 4, [47]). Let $0<s<\delta \leq 1$ and $T_{K} \in \operatorname{WSIO}(s, \delta)$. Then, $T_{K}(1) \in \dot{B}_{\infty}^{s, \infty}$. Moreover, there exists $C=C(s, \delta)$ such that: $\left\|T_{K}(1)\right\|_{\dot{B}_{\infty}^{s, \infty}} \leq C \kappa\left(T_{K}\right)$ where $\kappa\left(T_{K}\right)$ is the constant associated to $T_{K}$ in Definition 4 .

Lemma 32 (Remark 2, [47]). Let $1 \leq p, q<\infty, t<0$ and $s \in \mathbb{R}$. There exists $C=C(p, q, t, s)$ such that, for any $b \in \dot{B}_{\infty}^{s, \infty}, \pi_{b}$ is continuous from $\dot{F}_{p}^{t, q}$ to $\dot{F}_{p}^{t+s, q}$ and the following estimate holds:

$$
\forall f \in \dot{F}_{p}^{t, q},\left\|\pi_{b}(f)\right\|_{\dot{F}_{p}^{t+s, q}} \leq C\|b\|_{\dot{B}_{\infty}^{s, \infty}}\|f\|_{\dot{F}_{p}^{t, q}} .
$$

Lemma 33 (Lemma 2, [47]). Let $0<s<1$ and $0<\delta \leq 1$. Take $b \in \dot{B}_{\infty}^{s, \infty}$. Then, the operator $\pi_{b} \in$ $\operatorname{WSIO}(s, \delta)$. Moreover, there exists a constant $C(s)$ independent of $b$ such that, $\kappa\left(\pi_{b}\right) \leq C(s)\|b\|_{\dot{B}_{\infty}^{s, \infty}}$, where $\kappa\left(\pi_{b}\right)$ is the constant in Definition 4 associated to the operator $\pi_{b}$.

Combining these lemmas allows us to circumvent the $T(1)=0$ condition. Indeed:

Lemma 34. Let $0<s<\delta \leq 1$ and $1 \leq p, q<\infty$. Let $t \in \mathbb{R}$ be such that $-s<t<0$. There exists a constant $C$ such that, for $T_{K} \in \mathrm{WSIO}(s, \delta), T_{K}$ is continuous from $\dot{F}_{p}^{t, q}$ into $\dot{F}_{p}^{t+s, q}$ and we have:

$$
\forall f \in \dot{F}_{p}^{t, q}, \quad\left\|T_{K}(f)\right\|_{\dot{F}_{p}^{t+s, q}} \leq C \kappa\left(T_{K}\right)\|f\|_{\dot{F}_{p}^{t, q}},
$$

where $\kappa\left(T_{K}\right)$ is the constant associated to $T_{K}$ in Definition 4. 
Proof. Let $T_{K} \in \operatorname{WSIO}(s, \delta)$. Thanks to Lemma $31, T_{K}(1) \in \dot{B}_{\infty}^{s, \infty}$ and $\left\|T_{K}(1)\right\|_{\dot{B}_{\infty}^{s, \infty}} \lesssim \kappa\left(T_{K}\right)$. Thanks to Lemma $33, \pi_{T_{K}(1)} \in \operatorname{WSIO}(s, \delta)$ and $\kappa\left(\pi_{T_{K}(1)}\right) \lesssim \kappa\left(T_{K}\right)$. Hence, we can define $\tilde{T}_{K}:=$ $T_{K}-\pi_{T_{K}(1)}$ and $\tilde{T}_{K} \in \mathrm{WSIO}(s, \delta)$, with a constant $\kappa\left(\tilde{T}_{K}\right) \lesssim \kappa\left(T_{K}\right)$. Moreover, since $\pi_{b}(1)=b$ for any $b, \tilde{T}_{K}(1)=0$. Thanks to Lemma 32 , proving the continuity of $\tilde{T}_{K}$ is sufficient to obtain (A.19).

Let $a_{Q}$ be a smooth $t$-atom. We consider $m_{Q}=\tilde{T}_{K}\left(a_{Q}\right)$. The next step is to prove that $m_{Q}$ is almost a $(\delta, M)$ smooth $(t+s)$-molecule, with $M=n+s-\delta>n$. As noted above, since $t+s>0$, we only need to check (A.14) and (A.15). Indeed, lengthy computations and the essential condition $\tilde{T}_{K}(1)=0$ provide the existence of a constant $D$ independent on the atom $a_{Q}$ such that:

$$
\begin{gathered}
\left|m_{Q}(x)\right| \leq D \ell_{Q}^{s}\left(1+\ell_{Q}^{-1}\left|x-x_{Q}\right|\right)^{-M} \\
\left|m_{Q}(x)-m_{Q}\left(x^{\prime}\right)\right| \leq D \ell_{Q}^{s} \ell_{Q}^{-\delta}\left|x-x^{\prime}\right|^{\delta} \sup _{|z| \leq\left|x-x^{\prime}\right|}\left(1+\ell_{Q}^{-1}\left|z-\left(x-x_{Q}\right)\right|\right)^{-M} .
\end{gathered}
$$

Hence $\tilde{m}_{Q}:=D^{-1} \ell_{Q}^{-s} m_{Q}$ is a molecule. For examples of proof techniques to prove (A.20) and (A.21), we refer the reader to [44] and [47]. To conclude the proof, we use Lemma 29 and 30. For $f \in \dot{F}_{p}^{t, q}$, we write $f(x)=\sum_{Q} s_{Q} a_{Q}(x)$ and each $\tilde{m}_{Q}=D^{-1} \ell_{Q}^{-s} T_{K}\left(a_{Q}\right)$ is a molecule. Thus, thanks to Lemma 29 and Lemma 30 ,

$$
\begin{aligned}
\left\|T_{K}(f)\right\|_{\dot{F}_{p}^{t+s, q}} & =\left\|\sum_{Q}\left(D \ell_{Q}^{s} s_{Q}\right) \cdot m_{Q}(x)\right\|_{\dot{F}_{p}^{t+s, q}} \\
& \lesssim\left\|\left(\sum_{Q} \ell_{Q}^{-(t+s) q} D^{q} \ell_{Q}^{s q}\left|s_{Q}\right|^{q}\left|\chi_{Q}(x)\right|^{q}\right)^{1 / q}\right\|_{p} \\
& \lesssim\left\|\left(\sum_{Q} \ell_{Q}^{-t q}\left|s_{Q}\right|^{q}\left|\chi_{Q}(x)\right|^{q}\right)^{1 / q}\right\|_{p} \\
& \lesssim\|f\|_{\dot{F}_{p}^{t, q}} .
\end{aligned}
$$

Equation (A.22) concludes the proof.

Triebel-Lizorkin spaces offer a natural framework for atomic and molecular decompositions. Of course, setting $p=q=2$ in the results above also yields results for the more classical homogeneous Sobolev spaces $\dot{H}^{\alpha}$. Thus, Lemma 34 tells us that operators of $\operatorname{WSIO}(s, \delta)$ continuously map $\dot{H}^{t}$ into $\dot{H}^{t+s}$ for $-s<t<0$. In particular, this is valid for $s=1 / 2$ and $t=-1 / 4$.

\section{A.3 Kernels defined on bounded domains}

Most results involving singular integral operators concern kernels defined on the full space $\mathbb{R}^{n} \times \mathbb{R}^{n}$. Here, for finite time controllability, we need to adapt these results to a setting where the kernels are defined on squares, eg. $[0,1] \times[0,1]$. Atoms and molecules are localized functions. Thus, it would be possible to carry on the same proof as above for bounded domains, providing that the analogs of the representation lemmas 29 and 30 exist for Triebel-Lizorkin spaces on bounded domains. In this paragraph, we give another approach, which consists in proving that a kernel defined on a bounded domain can be extended while satisfying the same estimates.

Lemma 35. Let $n=1,0<s<1$ and $0<\delta \leq 1$. Consider a kernel $K$, defined and continuous on $\Omega_{1}=\left\{(x, y) \in[0,1]^{2}, x \neq y\right\}$, satisfying:

$$
\begin{gathered}
|K(x, y)| \leq \kappa|x-y|^{-1+s}, \\
\left|K\left(x^{\prime}, y\right)-K(x, y)\right| \leq \kappa\left|x^{\prime}-x\right|^{\delta}|x-y|^{-1+s-\delta}, \quad \text { for }\left|x^{\prime}-x\right| \leq \frac{1}{2}|x-y| \\
\left|K\left(x, y^{\prime}\right)-K(x, y)\right| \leq \kappa\left|y^{\prime}-y\right|^{\delta}|x-y|^{-1+s-\delta}, \quad \text { for }\left|y^{\prime}-y\right| \leq \frac{1}{2}|x-y|
\end{gathered}
$$

Then there exists a kernel $\bar{K}$ on $\mathbb{R} \times \mathbb{R}$, continuous on $\Omega$, such that: 
- $\bar{K}$ is an extension of $K:\left.\bar{K}\right|_{\Omega_{1}}=K$,

- $\bar{K}$ is a weakly singular integral operator of type $(s, \delta)$ on $\Omega$,

- $\bar{K}$ is associated a constant $\kappa(\bar{K}) \leq C \kappa(K)$, where $C$ is independent of $K, s$ and $\delta$.

Proof. We start by defining $\bar{K}(x, y)$ on the infinite strip $-1<y-x<1$. For $(x, y) \in \Omega_{1}$, we set $\bar{K}(x, y)=K(x, y)$. Outside of the initial square, we extend by continuity the values taken on the sides of the square and we choose an extension that is constant along all diagonal lines. Therefore, we define $\bar{K}(x, y)$ as:

$$
\begin{array}{r}
K(1+x-y, 1) \quad \text { for } \quad 1 \leq y, 0<y-x<1 \\
K(0, y-x) \text { for } \quad x \leq 0,0<y-x<1 \\
K(1,1+y-x) \text { for } 1 \leq x, 0<x-y<1 \\
K(x-y, 0) \text { for } y \leq 0,0<x-y<1 .
\end{array}
$$

Outside of the strip, we set:

$$
\begin{array}{ll}
\bar{K}(x, y)=K(0,1)|x-y|^{-1+s}, & \text { for } y-x \geq 1, \\
\bar{K}(x, y)=K(1,0)|x-y|^{-1+s}, & \text { for } x-y \geq 1 .
\end{array}
$$

This completes the definition of $\bar{K}$ on $\Omega$. By construction, it is easy to check that $\bar{K}$ is continuous on $\Omega$. By construction, $\bar{K}$ also satisfies (A.23) on $\Omega_{1}$, on the whole strip $-1 \leq y-x \leq 1$ thanks to (A.27) and on the half spaces $y-x \geq 1$ and $y-x \leq-1$ thanks to the decay chosen in (A.27).

The Hölder regularity estimates (A.24) and (A.25) are a little tougher. First, note that, by symmetry, one only needs to prove, for example, (A.24) on the half place $\mathcal{H}=\{(x, y) \in \mathbb{R} \times \mathbb{R}, \quad y-x>$ $0\}$. We write $\mathcal{H}=\tilde{\mathcal{H}} \cup \mathcal{H}_{1} \cup \mathcal{H}_{-} \cup \mathcal{H}_{+}$, where:

$$
\begin{aligned}
\tilde{\mathcal{H}} & =\{(x, y) \in \mathcal{H}, \quad y-x>1\}, \\
\mathcal{H}_{1} & =\{(x, y) \in \mathcal{H}, \quad 0 \leq x \text { and } y \leq 1\}, \\
\mathcal{H}_{+} & =\{(x, y) \in \mathcal{H}, \quad y-x \leq 1 \text { and } 1<y\}, \\
\mathcal{H}_{-} & =\{(x, y) \in \mathcal{H}, \quad y-x \leq 1 \text { and } x<0\} .
\end{aligned}
$$

Let $(x, y) \in \mathcal{H}$ and $\left(x^{\prime}, y\right) \in \mathcal{H}$ with $\left|x-x^{\prime}\right| \leq \frac{1}{2}|x-y|$. If both points belong to the same subdomain, then the Hölder regularity estimate in the $x$ direction for $\bar{K}$ is a direct consequence either of (A.27) on $\tilde{\mathcal{H}}$, of (A.26) on $\mathcal{H}_{ \pm}$and of the hypothesis on $K$ on $\mathcal{H}_{1}$. If the two points belong to different subdomains, we use a triangular inequality involving a point at the boundary separating the two subdomains. As an example of such a situation, if $x<0<x^{\prime}$ and $y<x+1$, then $(x, y) \in \mathcal{H}_{-}$and $\left(x^{\prime}, y\right) \in \mathcal{H}_{1}$. We have:

$$
\begin{aligned}
\left|\bar{K}(x, y)-\bar{K}\left(x^{\prime}, y\right)\right| & =\left|K(0, y-x)-K\left(x^{\prime}, y\right)\right| \\
& \leq|K(0, y-x)-K(0, y)|+\left|K(0, y)-K\left(x^{\prime}, y\right)\right| \\
& \leq \kappa|x|^{\delta}|x-y|^{-1+s-\delta}+\kappa\left|x^{\prime}\right|^{\delta}\left|x^{\prime}-y\right|^{-1+s-\delta} \\
& \leq 5 \kappa\left|x-x^{\prime}\right|^{\delta}|x-y|^{-1+s-\delta} .
\end{aligned}
$$

The last inequality comes from the fact that $\left|x^{\prime}\right|,|x| \leq\left|x-x^{\prime}\right|$ and $\left|x^{\prime}-y\right|^{-1+s-\delta} \leq 4|x-y|^{-1+s-\delta}$ for $\left|x-x^{\prime}\right| \leq \frac{1}{2}|x-y|$. The details of the other situations are left to the reader.

\section{References}

[1] Adi Adimurthi, Shyam Sundar Ghoshal, and G.D.Veerappa Gowda. Exact controllability of scalar conservation laws with strict convex flux. Submitted, 2012.

[2] Fabio Ancona and Andrea Marson. On the attainable set for scalar nonlinear conservation laws with boundary control. SIAM J. Control Optim., 36(1):290-312 (electronic), 1998.

[3] Hajer Bahouri, Jean-Yves Chemin, and Raphäl Danchin. Fourier analysis and nonlinear partial differential equations, volume 343 of Grundlehren der Mathematischen Wissenschaften [Fundamental Principles of Mathematical Sciences]. Springer, Heidelberg, 2011. 
[4] Karine Beauchard. Local controllability of a 1-D Schrödinger equation. J. Math. Pures Appl. (9), 84(7):851-956, 2005.

[5] Karine Beauchard and Jean-Michel Coron. Controllability of a quantum particle in a moving potential well. J. Funct. Anal., 232(2):328-389, 2006.

[6] Karine Beauchard, Jean-Michel Coron, and Holger Teismann. Minimal time for the bilinear control of Schrödinger equations. Systems Control Lett., 71:1-6, 2014.

[7] Karine Beauchard and Morgan Morancey. Local controllability of 1D Schrödinger equations with bilinear control and minimal time. Math. Control Relat. Fields, 4(2):125-160, 2014.

[8] Árpád Bényi, Diego Maldonado, and Virginia Naibo. What is ... a paraproduct? Notices Amer. Math. Soc., 57(7):858-860, 2010.

[9] Christian Berg, Jens Peter Reus Christensen, and Paul Ressel. Harmonic analysis on semigroups, volume 100 of Graduate Texts in Mathematics. Springer-Verlag, New York, 1984. Theory of positive definite and related functions.

[10] Jean-Michel Bony. Calcul symbolique et propagation des singularités pour les équations aux dérivées partielles non linéaires. Ann. Sci. École Norm. Sup. (4), 14(2):209-246, 1981.

[11] Roger Brockett. Controllability with quadratic drift. Math. Control Relat. Fields, 3(4):433-446, 2013.

[12] Pavol Brunovský. A classification of linear controllable systems. Kybernetika (Prague), 6:173-188, 1970.

[13] Alberto Pedero Calderón and Antoni Zygmund. On singular integrals. Amer. J. Math., 78:289$309,1956$.

[14] Alberto Pedro Calderón and Antoni Zygmund. On the existence of certain singular integrals. Acta Math., 88:85-139, 1952.

[15] Eduardo Cerpa. Exact controllability of a nonlinear Korteweg-de Vries equation on a critical spatial domain. SIAM J. Control Optim., 46(3):877-899 (electronic), 2007.

[16] Eduardo Cerpa and Emmanuelle Crépeau. Boundary controllability for the nonlinear Korteweg-de Vries equation on any critical domain. Ann. Inst. H. Poincaré Anal. Non Linéaire, 26(2):457-475, 2009.

[17] Marianne Chapouly. Global controllability of nonviscous and viscous Burgers-type equations. SIAM J. Control Optim., 48(3):1567-1599, 2009.

[18] Jean-Michel Coron. On the small-time local controllability of a quantum particle in a moving one-dimensional infinite square potential well. C. R. Math. Acad. Sci. Paris, 342(2):103-108, 2006 .

[19] Jean-Michel Coron. Control and nonlinearity, volume 136 of Mathematical Surveys and Monographs. American Mathematical Society, Providence, RI, 2007.

[20] Jean-Michel Coron. Some open problems on the control of nonlinear partial differential equations. In Perspectives in nonlinear partial differential equations, volume 446 of Contemp. Math., pages 215-243. Amer. Math. Soc., Providence, RI, 2007.

[21] Jean-Michel Coron and Emmanuelle Crépeau. Exact boundary controllability of a nonlinear KdV equation with critical lengths. J. Eur. Math. Soc. (JEMS), 6(3):367-398, 2004.

[22] Jean-Michel Coron and Sergio Guerrero. Singular optimal control: a linear 1-D parabolichyperbolic example. Asymptot. Anal., 44(3-4):237-257, 2005.

[23] Guy David and Jean-Lin Journé. A boundedness criterion for generalized Calderón-Zygmund operators. Ann. of Math. (2), 120(2):371-397, 1984. 
[24] Jesús Ildefondo Diaz. Obstruction and some approximate controllability results for the Burgers equation and related problems. In Control of partial differential equations and applications (Laredo, 1994), volume 174 of Lecture Notes in Pure and Appl. Math., pages 63-76. Dekker, New York, 1996.

[25] Enrique Fernández-Cara and Sergio Guerrero. Null controllability of the Burgers system with distributed controls. Systems Control Lett., 56(5):366-372, 2007.

[26] Michael Frazier and Björn Jawerth. Decomposition of Besov spaces. Indiana Univ. Math. J., 34(4):777-799, 1985.

[27] Michael Frazier and Björn Jawerth. The $\phi$-transform and applications to distribution spaces. In Function spaces and applications (Lund, 1986), volume 1302 of Lecture Notes in Math., pages 223-246. Springer, Berlin, 1988.

[28] Michael Frazier and Björn Jawerth. A discrete transform and decompositions of distribution spaces. J. Funct. Anal., 93(1):34-170, 1990.

[29] Michael Frazier, Björn Jawerth, and Guido Weiss. Littlewood-Paley theory and the study of function spaces, volume 79 of CBMS Regional Conference Series in Mathematics. Published for the Conference Board of the Mathematical Sciences, Washington, DC; by the American Mathematical Society, Providence, RI, 1991.

[30] Andrei Fursikov and Oleg Imanuvilov. On controllability of certain systems simulating a fluid flow. In Flow control (Minneapolis, MN, 1992), volume 68 of IMA Vol. Math. Appl., pages 149-184. Springer, New York, 1995.

[31] Andrei Fursikov and Oleg Imanuvilov. Controllability of evolution equations, volume 34 of Lecture Notes Series. Seoul National University Research Institute of Mathematics Global Analysis Research Center, Seoul, 1996.

[32] Sergio Guerrero and Oleg Imanuvilov. Remarks on global controllability for the Burgers equation with two control forces. Ann. Inst. H. Poincaré Anal. Non Linéaire, 24(6):897-906, 2007.

[33] Thierry Horsin. On the controllability of the Burgers equation. ESAIM Control Optim. Calc. Var., 3:83-95 (electronic), 1998.

[34] Jacques-Louis Lions. Quelques méthodes de résolution des problèmes aux limites non linéaires. Dunod, 1969.

[35] Frédéric Marbach. Small time global null controllability for a viscous Burgers' equation despite the presence of a boundary layer. J. Math. Pures Appl. (9), 102(2):364-384, 2014.

[36] James Mercer. Functions of positive and negative type, and their connection with the theory of integral equations. Philosophical Transactions of the Royal Society of London. Series A, Containing Papers of a Mathematical or Physical Character, 209:pp. 415-446, 1909.

[37] Boris Vasil'evich Pal'cev. Asymptotic behavior of the spectrum and eigenfunctions of convolution operators on a finite interval with the kernel having a homogeneous Fourier transform. Dokl. Akad. Nauk SSSR, 218:28-31, 1974.

[38] Amnon Pazy. Semigroups of linear operators and applications to partial differential equations, volume 44 of Applied Mathematical Sciences. Springer-Verlag, New York, 1983.

[39] Vincent Perrollaz. Exact controllability of scalar conservation laws with an additional control in the context of entropy solutions. Submitted, 2011.

[40] Murray Rosenblatt. Some results on the asymptotic behavior of eigenvalues for a class of integral equations with translation kernels. J. Math. Mech., 12:619-628, 1963.

[41] Lionel Rosier. Exact boundary controllability for the Korteweg-de Vries equation on a bounded domain. ESAIM Control Optim. Calc. Var., 2:33-55 (electronic), 1997.

[42] Elias Menachem Stein. Singular integrals and differentiability properties of functions. Princeton Mathematical Series, No. 30. Princeton University Press, Princeton, N.J., 1970. 
[43] Héctor Sussmann. Lie brackets and local controllability: a sufficient condition for scalar-input systems. SIAM J. Control Optim., 21(5):686-713, 1983.

[44] Rodolfo Torres. Boundedness results for operators with singular kernels on distribution spaces. Mem. Amer. Math. Soc., 90(442):viii+172, 1991.

[45] Emmanuel Trélat. Contrôle optimal. Mathématiques Concrètes. [Concrete Mathematics]. Vuibert, Paris, 2005. Théorie \& applications. [Theory and applications].

[46] Harold Widom. Asymptotic behavior of the eigenvalues of certain integral equations. Trans. Amer. Math. Soc., 109:278-295, 1963.

[47] Abdellah Youssfi. Regularity properties of singular integral operators. Studia Math., 119(3):199$217,1996$. 